\title{
Wagner Equation Predicting Entire Curve for Pure Fluids from Limited VLE Data: Critical and Normal Boiling Points \& Acentric Factor
}

\author{
T.T. Nichols ${ }^{1}$, V.P. Utgikar ${ }^{2 *}$ \\ ${ }^{1,2}$ Department of Chemical \& Materials Engineering, University of Idaho, Moscow, Idaho, USA \\ E-mail: ${ }^{1}$ toddnich2@gmail.com, ${ }^{2}$ vutgikar@uidaho.edu
}

Received 23 May 2018, Accepted 14 August 2018

\begin{abstract}
Wagner constants are estimated from VLE predictions based solely on the critical and normal boiling points, and acentric factor. The predictions come from the standard forms of 1) Riedel and 2) Ambrose-Walton, 3) a modified Riedel equation resulting from forcing the value of $\mathrm{K}$ to provide zero error at the reduced temperature of 0.7 , and 4 ) a modified Ambrose-Walton equation resulting from using a pseudo acentric factor that provides zero error at the normal boiling point. Reduced vapor pressure values obtained using these Wagner constants are compared to those generated by using literature-reported Wagner constants that are applicable for the entire two-phase VLE curve for 72 species. It is shown that the Wagner equation parameterized using VLE predictions from such correlations can provide better accuracy than the underlying source correlations themselves, and it can exhibit less error than a parameterization using a limited interval of experimental data.
\end{abstract}

Keywords: Vapor-liquid equilibrium; Wagner equation; Riedel equation; Ambrose-Walton equation.

\section{Introduction}

A primary goal of our ongoing research is to provide a practitioner with better approaches to predict entire-curve VLE for pure substances with as little required data as possible. The Wagner equation is typically used to describe VLE over the entire two-phase region because it has been shown to be successful at accurately representing phase equilibrium along the entire co-existence curve for many substances [1]-[5]. The Wagner equation is generally not considered to be predictive because the constants are not known a priori; being unique for each substance, the four constants are usually determined by data-fitting algorithms.

Several researchers have used limited data to estimate Wagner constants that were then used to extend VLE predictions in one direction, either interpolation upward to the critical point [6]-[9] or extrapolation downward to the triple point [3], [10]-[12]. Researchers have consistently found alcohols to be problematic and to respond differently than other species to their parameterization approaches.

In his study of normal light hydrocarbons, Thodos [13] showed that pure species tend to have an elongated S-shaped curve when logarithmic vapor pressure is plotted versus the reciprocal thermodynamic temperature. The plot has curvature at low pressures, becomes more linear after the normal boiling point, and the plot undergoes a reversal of curvature at moderate pressures as the critical point is approached. Waring [14] defined the S-shaped curve in more exact mathematical form in terms of heat of vaporization and compressibility, noting that the upper inflection point usually occurred at a reduced temperature between 0.8 and 0.85 , and recommended that the presence of this inflection point be used as a qualitative fit-of-form test for VLE predictive methods.
A broader range for the location of the inflection point has subsequently been determined [7]. McGarry [8] used the inflection point as one of his three fitting constraints, attempted to correlate its location to the temperature of a species' normal boiling point, and noted that species with a normal boiling point below $50 \mathrm{~K}$ did not exhibit the inflection point. The presence of the inflection point is implicit in the Riedel equation [15], and the AmbroseWalton equation has good success at predicting its location [16].

Our work is focused on further exploring the ability of the Wagner equation to become predictive for the entire twophase region. It has been shown that a single set of four VLE points of good precision from limited data can be used to estimate Wagner constants that can generally provide VLE predictions over the entire two-phase curve for pure substances with good accuracy [17].

The impact of data repeatability/reproducibility disagreement was subsequently investigated by using entirecurve Wagner analytics for 55 species to represent the "best" values and Antoine analytics to represent single sets of limited-range experimental data [18]. The results led to several conclusions: 1) VLE data in the literature can deviate significantly from "best" values; 2) Wagner constants estimated from analytic values from another correlation of different form can have greater predictive power than that of the source correlation; 3 ) the fully-determined approach to estimate Wagner constants from Antoine analytics failed the qualitative Waring fit-of-form test for several species; and 4) Wagner constants estimated from Antoine analytics generally had superior predictive power for alcohols, the Riedel equation was best for organic acids, and the AmbroseWalton equation had least error for the remaining species (mostly normal, and a few polar). 
Given the relative good performance of Riedel and Ambrose-Walton equations for species other than alcohols, their ability to satisfy the Waring fit-of-form test, and their functional form being different from that of the Wagner equation, the goal of this paper is to determine if Wagner constants estimated using predictions from the Riedel and Ambrose-Walton correlations can result in superior predictive power while also satisfying the qualitative Waring fit-of-form test.

The results of using predicted VLE values from standard and modified versions of the Riedel and Ambrose-Walton equations to estimate Wagner constants are presented. The predictive error of these Wagner constants is compared first with that of the source equations and second with that of Wagner constants estimated from limited-range Antoine analytics. For many species only the critical and normal boiling points and acentric factor are given in technical handbooks - thus, the benefit of using Riedel and AmbroseWalton as the source correlations. This paper presents a new approach for using only these three known vapor pressure data points to predict VLE over the entire two-phase curve. The results presented here are relevant to the research of predictive correlation development and to engineers tasked with predicting VLE for a species when only these three data points are available.

\section{Approach}

Our approach has two notable elements. First, a fourpoint, fully-determined solution rather than a complex overdetermined solution is used. That significant predictive success can arise from a relatively simple, algebraic parameterization of the Wagner equation has practical utility as it can potentially reduce computational burden. Second is the hypothesis that the Wagner equation parameterized from VLE predictions can have better predictive error than the source correlations themselves, and that such parameterizations can provide less error than parameterizations using limited data intervals. The concept of having source predictive correlations utilizing only a few experimental anchor points to provide pseudo data with which another predictive correlation is parameterized and applied to the entire two-phase curve proves to be insightful though appearing to be counter intuitive.

The generally preferred form of the Wagner equation [5] is used in our work,

Ln $P_{v, r}=\left(a \tau+b \tau^{1.5}+c \tau^{2.5}+d \tau^{5}\right) / T_{r}$

$L n$ refers to natural logarithm, $P$ refers to pressure, and $T$ represents thermodynamic temperature. The subscript $v$ indicates the pressure corresponds to the vapor-liquid equilibrium curve, and $r$ indicates that the parameter is reduced with respect to the critical point (i.e., $T_{r}=T / T_{c}$ and $P_{v, r}=P_{v} / P_{c}$, where the subscript $c$ refers to the critical point). The parameter $\tau$ equals $1-T_{r}$.

Wagner constants found in the literature that are applicable to the entire two-phase VLE curve are referred to here as "entire-curve" constants. Vapor pressures calculated from such entire-curve Wagner constants are referred to as "entire-curve analytic" data and are used as the reference standard, the "best" values, for error comparison.

In the absence of an accurate vapor pressure data/correlation for a temperature interval of interest, it is recommend that the Riedel or Ambrose-Walton methods be used to predict VLE at low temperatures and a tworeference-fluid or Riedel method for polar substances in the reduced temperature range 0.5-1.0 [5]. This provides additional support for using the Riedel and Ambrose-Walton equations as the sources of VLE predictions in our fullydetermined approach to estimate Wagner constants.

The standard form of Ambrose-Walton used in this work is given by [5], [19]

$\operatorname{Ln} P_{v, r}=f^{(0)}+\omega f^{(1)}+\omega^{2} f^{(2)}$

where

$f^{(0)}=\frac{-5.97616 \tau+1.29874 \tau^{1.5}-0.60394 \tau^{2.5}-1.06841 \tau^{5}}{T_{r}}$

$f^{(1)}=\frac{-5.03365 \tau+1.11505 \tau^{1.5}-5.41217 \tau^{2.5}-7.46628 \tau^{5}}{T_{r}}$

$f^{(2)}=\frac{-0.64771 \tau+2.41539 \tau^{1.5}-4.26979 \tau^{2.5}+3.25259 \tau^{5}}{T_{r}}$

The standard Riedel equation has the normal boiling point as an anchor and is given by (as cited in Ref. [5])

$\operatorname{Ln} P_{v, r}=A^{+}-\frac{B^{+}}{T_{r}}+C^{+} L n T_{r}+D^{+} T_{r}^{6}$

where

$A^{+}=-35 Q ; B^{+}=-36 Q ; C^{+}=42 Q+\alpha_{c} ;$

$D^{+}=-Q$

$Q=K\left(3.758-\alpha_{c}\right)$

$\propto_{c}=\frac{3.758 K \psi_{b}-\operatorname{Ln} P_{v, r, b}}{K \psi_{b}-\operatorname{Ln} T_{r, b}}$

$\psi_{b}=-35+\frac{36}{T_{r, b}}+42 \operatorname{Ln} T_{r . b}-T_{r, b}^{6}$

The subscript $b$ on $\psi$ indicates that the parameter is based on the reduced temperature of the normal boiling point. $K$ has the value of 0.0838 , except for organic acids and alcohols, in which case the linear relationships of Vetere[2], [20] are used,

$K=-0.120+0.025 h$

and

$K=0.373-0.030 h$

respectively, where

$h=-T_{r, b} \frac{\operatorname{Ln} P_{v, r, b}}{1-T_{r, b}}$

The Wagner constants and physical properties of the 72 species studied are given in Table 1. Although the dipole moment is not used as a mathematical input in our approach, it is included in the table to provide an estimate of polarity. The significant digits displayed in Table 1 are those given by the referenced sources. The group of 72 species studied in our work include 22 alcohols, 9 organic acids, 6 other polar species, and 35 normal substances (the quantum gases hydrogen and helium are included in the count of normal substances).

Besides using their standard forms, a modified form of Riedel is created by forcing the value of $K$ to provide zero error at the reduced temperature of 0.7 , and a modified Ambrose-Walton is created by using a pseudo acentric factor that provides zero error at the normal boiling point.

\subsection{Modification of Riedel Equation}

The standard Riedel equation uses the normal boiling point as an anchor point, but any other saturation point can 
become a secondary anchor by using it to back calculate the value of $K$. Inverting Eq. (3) and substituting Eqs. (3a) and (3b) for $A^{+}, B^{+}, C^{+}, D^{+}$, and $Q$ gives a substance-specific relationship for $K$ based upon an additional saturation point,

$K=\frac{L n P_{v, r, S A}-\alpha_{c} L n T_{r, S A}}{\left(3.758-\alpha_{c}\right) \psi_{S A}}$

The subscript " $S A$ " has been added to indicate that a variable or parameter pertains to the secondary anchor point. The parameter $\psi_{S A}$ has the same functional form as that of $\psi_{b}$ and is given by

$\psi_{S A}=-35+\frac{36}{T_{r, S A}}+42 \operatorname{Ln} T_{r, S A}-T_{r, S A}^{6}$

The parameter $\alpha_{c}$ is dependent upon $K$, so Eq. (3c) has to be substituted for $\alpha_{c}$ into Eq. (4) to arrive at the final relationship for the substance-dependent K,

$K=\frac{\left(\operatorname{Ln} P_{v, r, b}\right)\left(L n T_{r, S A}\right)-\left(\operatorname{Ln} P_{v, r, S A}\right)\left(\operatorname{Ln} T_{r, b}\right)}{K^{\prime}}$

where the denominator in the above equation is given by

$$
\begin{aligned}
K^{\prime}= & 3.758\left[\left(\operatorname{Ln} T_{r, S A}\right) \psi_{b}-\left(\operatorname{Ln} T_{r, b}\right) \psi_{S A}\right]+ \\
& \left(\operatorname{Ln} P_{v, r, b}\right) \psi_{S A}-\left(\operatorname{Ln} P_{v, r, S A}\right) \psi_{b}
\end{aligned}
$$

Using the reduced temperature of 0.7 for the secondary anchor point, Eq. (5) has the potential to result in a negative value for $K$ because of round off error if the normal boiling point reduced temperature is very close to 0.7 (only three significant digits right of the decimal point are used for the acentric factor). Such is the situation for one of the 72 species, butanoic acid, where the difference between $T_{r, b}$ and 0.7 is only 0.00011 , in which case the Vetere value of $K$ given by Eq. (3e) is retained.

The notation Riedel $(\omega)$, or the short hand $R(\omega)$, is used to represent using Eq. (4b) and the acentric factor to determine the value of $K$ : the value calculated from Eq. (4b) is used if positive, and the Vetere value of $K$ is used if the value calculated from Eq. (6) is negative, in which case the standard Riedel and $R(\omega)$ are equivalent.

\subsection{Modified Ambrose-Walton Equation}

Unlike the Riedel equation with its $K$ parameter, the Ambrose-Walton relationship with its twelve coefficients doesn't have a single parameter that can be methodically adjusted to provide an additional universal anchor point. We choose to swap the normal boiling point with the reduced temperature of 0.7 as the anchor point. The anchor point can be changed by simply using the acentric factor estimated from the normal boiling point. The Ambrose-Walton is quadratic in the acentric factor; thus, the acentric factor can be estimated from any saturation point by

$\omega=\frac{-f^{(1)} \pm \sqrt{\left(f^{(1)}\right)^{2}-4 f^{(2)}\left(f^{(0)}-L n P_{v, r}\right)}}{2 f^{(2)}}$

Using the normal boiling point, values calculated from Eq. (5) using the negative square root in the numerator were compared with the acentric factor values given in Ref. [5] for 313 species. The produced acentric values have an average absolute deviation of 0.004 , with 10 species having a deviation greater than 0.02. The notation AmbroseWalton $\left(T_{b}\right)$, or the short hand $\mathrm{A}-\mathrm{W}\left(T_{b}\right)$, is used to represent using in Eq. (2) the value of the acentric factor given by Eq. (5) using the normal boiling point and the negative square root. Although Ref. [5] notes that for most species the $\omega^{2}$ term in the Ambrose-Walton equation is negligible when using it to estimate the acentric factor from the normal boiling point vapor pressure, we choose to use the quadratic because it is easy to calculate and it removes any potential error arising from a linear estimate.

\subsection{Algebraic Solution to Wagner Equation for Fully- Determined Case}

Given that the Wagner equation has four parameters, an algebraic solution for each parameter can be obtained with only four points. The resulting $F_{w, j}$ function to estimate Wagner constants from four data points (i.e., the fullydetermined case) is given by

$$
\begin{aligned}
F_{w, j}= & \eta_{j 1} \operatorname{Ln} P_{v, r, T_{r 1}}+\eta_{j 2} \operatorname{Ln} P_{v, r, T_{r 2}}+ \\
& \eta_{j 3} \operatorname{Ln} P_{v, r, T r 3}+\eta_{j 4} \operatorname{Ln} P_{v, r, T r 4}
\end{aligned}
$$

The subscript $j$ refers to the Wagner constant $(a, b, c$, or $d$ ). The reduced temperature subscript on $\operatorname{Ln} P_{v, r}$ and the value of the " $i$ " subscript in the $\eta_{j i}$ terms indicate the corresponding data point of the four selected VLE data. The only arguments of the universal $F_{w, j}$ functions are a substance's four $L n P_{v, r}$ values. The sixteen $\eta_{j i}$ coefficients (4 for each of the 4 Wagner constants) are functions only of the four reduced temperatures and are the same for all species for any chosen set of four reduced temperatures. The algebraic definitions of the $\eta_{j i}$ terms have been given elsewhere [17].

\section{Discussion \\ 3.1 Hypothesized Performance of Modified Correlations Relative to Standard Forms}

It is hypothesized that the different anchor point in the modified forms will improve the predictive accuracy on the part of the two-phase VLE curve in which the new anchor point resides. With a second anchor point at the reduced temperature of 0.7 , Riedel $(\omega)$ is expected to perform better than the standard Riedel below the normal boiling point for the case when $T_{r, b}>0.7$ and above the normal boiling point when $T_{r, b}<0.7$. Alternatively, with the acentric factor defined by the normal boiling point, $\mathrm{A}-\mathrm{W}\left(T_{b}\right)$ is expected to perform better than the standard Ambrose-Walton below the normal boiling point for the case when $T_{r, b}<0.7$ because the anchor point is lower down the VLE curve than is the reduced temperature 0.7 . Similarly, $\mathrm{A}-\mathrm{W}\left(T_{b}\right)$ is expected to perform better than the standard Ambrose-Walton above the normal boiling point for the case when $T_{r, b}>0.7$ because the anchor point is higher up the VLE curve than is the reduced temperature 0.7 . The expected relative performance is summarized in Table 2. There is no hypothesized relative predictive accuracy for the part of the two-phase VLE curve in which the anchor point does not reside; thus, the blank cells in Table 2 . 
Table 1. Wagner Constants and Physical Properties.

\begin{tabular}{|c|c|c|c|c|c|c|c|c|c|c|}
\hline \multirow[b]{2}{*}{ Species } & \multicolumn{4}{|c|}{ Wagner Constants } & \multicolumn{6}{|c|}{ Physical Properties } \\
\hline & $a$ & $b$ & $c$ & $d$ & $T_{f}(\mathrm{~K})$ & $T_{b}(\mathrm{~K})$ & $T_{c}(\mathrm{~K})$ & $P_{c}$ (bar) & $\omega$ & $\begin{array}{c}\mu \\
\text { (Debye) }\end{array}$ \\
\hline 2-Methyl propanoic acid $\left(\mathrm{C}_{4} \mathrm{H}_{8} \mathrm{O}_{2}\right)$ & -8.53258 & 1.30605 & -5.2242 & -2.05813 & 227.05 & 427.57 & 605.00 & 37.00 & 0.618 & 1.3 \\
\hline 3-Methyl butanoic acid $\left(\mathrm{C}_{5} \mathrm{H}_{10} \mathrm{O}_{2}\right)$ & -8.67381 & 1.62939 & -6.51756 & -2.08757 & 243.85 & 449.68 & 629.00 & 34.00 & 0.651 & \\
\hline Acetic Acid $\left(\mathrm{C}_{2} \mathrm{H}_{4} \mathrm{O}_{2}\right)$ & -8.29430 & 0.97928 & -0.21745 & -5.72367 & 289.77 & 391.04 & 592.71 & 57.86 & 0.459 & 1.3 \\
\hline Butanoic acid $\left(\mathrm{C}_{4} \mathrm{H}_{8} \mathrm{O}_{2}\right)$ & -8.42953 & 1.34333 & -5.37332 & -2.74438 & 267.97 & 436.87 & 624.00 & 40.30 & 0.600 & 1.5 \\
\hline Decanoic acid $\left(\mathrm{C}_{10} \mathrm{H}_{20} \mathrm{O}_{2}\right)$ & -9.07060 & 2.77535 & -11.1014 & -2.43545 & 305.15 & 541.92 & 726.00 & 22.30 & 0.749 & \\
\hline Formic acid $\left(\mathrm{CH}_{2} \mathrm{O}_{2}\right)$ & -7.24917 & 0.44255 & -0.35558 & -0.96906 & 281.50 & 374.04 & 588.00 & 58.07 & 0.316 & 1.5 \\
\hline Octanoic acid $\left(\mathrm{C}_{8} \mathrm{H}_{16} \mathrm{O}_{2}\right)$ & -9.04015 & 2.16529 & -8.66117 & -4.69516 & 289.45 & 512.01 & 695.00 & 26.40 & 0.734 & \\
\hline Pentanoic acid $\left(\mathrm{C}_{5} \mathrm{H}_{10} \mathrm{O}_{2}\right)$ & -8.76701 & 1.54990 & -6.19961 & -4.21927 & 239.45 & 459.31 & 643.00 & 35.80 & 0.670 & \\
\hline Propanoic acid $\left(\mathrm{C}_{3} \mathrm{H}_{6} \mathrm{O}_{2}\right)$ & -8.14882 & 0.79590 & -3.1836 & -3.81338 & 252.31 & 414.31 & 604.00 & 45.30 & 0.539 & 1.5 \\
\hline 1-Butanol $\left(\mathrm{C}_{4} \mathrm{H}_{10} \mathrm{O}\right)$ & -8.40615 & 2.23010 & -8.2486 & -0.7110 & 183.35 & 390.88 & 563.05 & 44.24 & 0.591 & 1.8 \\
\hline 1-Decanol $\left(\mathrm{C}_{10} \mathrm{H}_{22} \mathrm{O}\right)$ & -9.75478 & 4.18634 & -7.0572 & -15.980 & 280.05 & 504.25 & 689.00 & 24.10 & 0.629 & 1.8 \\
\hline 1-Dodecanol $\left(\mathrm{C}_{12} \mathrm{H}_{26} \mathrm{O}\right)$ & -9.91901 & 3.61884 & -5.8537 & -18.204 & 297.1 & 537.79 & 720.00 & 20.80 & 0.684 & \\
\hline 1-Eicosanol $\left(\mathrm{C}_{20} \mathrm{H}_{42} \mathrm{O}\right)$ & -11.23154 & 3.66900 & -7.0775 & -14.321 & 339.00 & 647.69 & 809.00 & 13.00 & 0.954 & \\
\hline 1-Heptadecanol $\left(\mathrm{C}_{17} \mathrm{H}_{36} \mathrm{O}\right)$ & -10.73125 & 3.55515 & -6.3591 & -15.696 & 327.00 & 611.12 & 780.00 & 15.00 & 0.853 & \\
\hline 1-Heptanol $\left(\mathrm{C}_{7} \mathrm{H}_{16} \mathrm{O}\right)$ & -9.68778 & 5.35716 & -10.1672 & -8.0100 & 239.20 & 449.81 & 632.50 & 31.35 & 0.580 & 1.7 \\
\hline 1-Hexadecanol $\left(\mathrm{C}_{16} \mathrm{H}_{34} \mathrm{O}\right)$ & -10.54087 & 3.47260 & -6.0770 & -15.939 & 322.45 & 597.53 & 770.00 & 16.10 & 0.818 & \\
\hline 1-Hexanol $\left(\mathrm{C}_{6} \mathrm{H}_{14} \mathrm{O}\right)$ & -9.49034 & 5.13288 & -10.5817 & -5.1540 & 229.20 & 430.44 & 610.70 & 34.70 & 0.575 & 1.8 \\
\hline 1-Nonanol $\left(\mathrm{C}_{9} \mathrm{H}_{20} \mathrm{O}\right)$ & -9.91542 & 5.13670 & -8.8075 & -12.497 & 268.15 & 486.52 & 671.50 & 26.30 & 0.610 & 1.7 \\
\hline 1-Octadecanol $\left(\mathrm{C}_{18} \mathrm{H}_{38} \mathrm{O}\right)$ & -10.91637 & 3.57835 & -6.6199 & -15.060 & 331.00 & 623.57 & 790.00 & 14.40 & 0.892 & \\
\hline 1-Octanol $\left(\mathrm{C}_{8} \mathrm{H}_{18} \mathrm{O}\right)$ & -10.01437 & 5.90629 & -10.4026 & -9.0480 & 257.65 & 468.33 & 652.50 & 28.60 & 0.594 & 2.0 \\
\hline 1-Pentanol $\left(\mathrm{C}_{5} \mathrm{H}_{12} \mathrm{O}\right)$ & -8.98005 & 3.91624 & -9.9081 & -2.1910 & 194.25 & 411.16 & 588.15 & 39.09 & 0.579 & 1.7 \\
\hline 2-Butanol $\left(\mathrm{C}_{4} \mathrm{H}_{10} \mathrm{O}\right)$ & -8.09820 & 1.64406 & -7.4900 & -5.27355 & 158.5 & 372.66 & 536.01 & 41.98 & 0.577 & 1.7 \\
\hline 2-Ethyl-1-hexanol $\left(\mathrm{C}_{8} \mathrm{H}_{18} \mathrm{O}\right)$ & -9.61812 & 5.17861 & -9.1144 & -11.004 & 203.20 & 457.77 & 640.50 & 27.99 & 0.558 & 1.8 \\
\hline 2-Octanol $\left(\mathrm{C}_{8} \mathrm{H}_{18} \mathrm{O}\right)$ & -9.37352 & 4.73760 & -8.3382 & -11.646 & 241.15 & 453.03 & 638.00 & 28.90 & 0.534 & 1.6 \\
\hline Benzyl alcohol $\left(\mathrm{C}_{7} \mathrm{H}_{8} \mathrm{O}\right)$ & -7.29099 & 1.17084 & -4.7167 & -5.5300 & 257.80 & 478.46 & 715.00 & 43.00 & 0.390 & 1.7 \\
\hline Cyclohexanol $\left(\mathrm{C}_{6} \mathrm{H}_{12} \mathrm{O}\right)$ & -7.12838 & 1.40189 & -5.60756 & -9.57158 & 297.65 & 433.94 & 650.00 & 42.60 & 0.370 & 1.7 \\
\hline Ethanol $\left(\mathrm{C}_{2} \mathrm{H}_{6} \mathrm{O}\right)$ & -8.68587 & 1.17831 & -4.8762 & 1.5880 & 159.05 & 351.80 & 513.92 & 61.32 & 0.643 & 1.7 \\
\hline Isopropyl alcohol $\left(\mathrm{C}_{3} \mathrm{H}_{8} \mathrm{O}\right)$ & -8.73656 & 2.16240 & -8.70785 & 4.77927 & 183.65 & 355.39 & 508.30 & 47.62 & 0.665 & 1.7 \\
\hline Methanol $\left(\mathrm{CH}_{4} \mathrm{O}\right)$ & -8.63571 & 1.17982 & -2.4790 & -1.0240 & 175.49 & 337.69 & 512.64 & 80.92 & 0.564 & 1.7 \\
\hline Propanol $\left(\mathrm{C}_{3} \mathrm{H}_{8} \mathrm{O}\right)$ & -8.53706 & 1.96214 & -7.6918 & 2.9450 & 147.00 & 370.93 & 536.78 & 51.68 & 0.620 & 1.7 \\
\hline Tert-butanol $\left(\mathrm{C}_{4} \mathrm{H}_{10} \mathrm{O}\right)$ & -8.47927 & 2.47845 & -9.27918 & -2.53992 & 298.55 & 355.49 & 506.20 & 39.73 & 0.613 & 1.7 \\
\hline Acetone $\left(\mathrm{C}_{3} \mathrm{H}_{6} \mathrm{O}\right)$ & -7.55098 & 1.60784 & -1.9944 & -3.2002 & 178.50 & 329.22 & 508.10 & 47.02 & 0.307 & 2.9 \\
\hline Cyclopentanone $\left(\mathrm{C}_{5} \mathrm{H}_{8} \mathrm{O}\right)$ & -7.36589 & 1.54092 & -2.28143 & -3.0514 & 222.5 & 403.72 & 624.50 & 46.00 & 0.288 & 3.0 \\
\hline Methyl isobutyl ketone $\left(\mathrm{C}_{6} \mathrm{H}_{12} \mathrm{O}\right)$ & -7.70040 & 1.69968 & -2.80448 & -3.81623 & 189.15 & 389.15 & 574.60 & 32.70 & 0.351 & 2.8 \\
\hline Benzene $\left(\mathrm{C}_{6} \mathrm{H}_{6}\right)$ & -7.01433 & 1.55256 & -1.8479 & -3.7130 & 278.68 & 353.24 & 562.16 & 48.98 & 0.209 & 0.0 \\
\hline Ethylbenzene $\left(\mathrm{C}_{8} \mathrm{H}_{10}\right)$ & -7.53139 & 1.75439 & -2.42012 & -3.57146 & 178.18 & 409.36 & 617.20 & 36.00 & 0.302 & 0.4 \\
\hline Naphthalene $\left(\mathrm{C}_{10} \mathrm{H}_{8}\right)$ & -7.61444 & 1.91553 & -2.5075 & -3.2300 & 351.35 & 491.16 & 748.40 & 40.50 & 0.304 & 0.0 \\
\hline Toluene $\left(\mathrm{C}_{7} \mathrm{H}_{8}\right)$ & -7.31600 & 1.59425 & -1.93165 & -3.72220 & 178.16 & 383.79 & 591.80 & 41.06 & 0.264 & 0.4 \\
\hline Pentafluorobenzene $\left(\mathrm{C}_{6} \mathrm{HF}_{5}\right)$ & -7.86799 & 1.71659 & -2.53582 & -4.59937 & 225.85 & 358.89 & 530.97 & 35.37 & 0.374 & \\
\hline Pentafluorotoluene $\left(\mathrm{C}_{7} \mathrm{H}_{3} \mathrm{~F}_{5}\right)$ & -8.08717 & 1.76131 & -2.72838 & -4.13797 & 243.35 & 390.65 & 566.52 & 31.24 & 0.415 & \\
\hline m-Xylene $\left(\mathrm{C}_{8} \mathrm{H}_{10}\right)$ & -7.67717 & 1.80240 & -2.47745 & -3.66068 & 225.28 & 412.34 & 617.05 & 35.38 & 0.326 & 0.3 \\
\hline o-Xylene $\left(\mathrm{C}_{8} \mathrm{H}_{10}\right)$ & -7.60491 & 1.75383 & -2.27531 & -3.73771 & 247.97 & 417.59 & 630.33 & 37.35 & 0.312 & 0.5 \\
\hline p-Xylene $\left(\mathrm{C}_{8} \mathrm{H}_{10}\right)$ & -7.71694 & 1.89119 & -2.39695 & -3.63026 & 286.41 & 411.53 & 616.23 & 35.16 & 0.322 & 0.1 \\
\hline Acetic Anhydride $\left(\mathrm{C}_{4} \mathrm{H}_{6} \mathrm{O}_{3}\right)$ & -8.35130 & 1.89050 & -2.8357 & -5.1156 & 199.00 & 412.69 & 606.00 & 40.00 & 0.456 & 3.0 \\
\hline Butane $\left(\mathrm{C}_{4} \mathrm{H}_{10}\right)$ & -7.01763 & 1.67770 & -1.9739 & -2.1720 & 134.79 & 272.66 & 425.25 & 37.92 & 0.199 & 0.0 \\
\hline Diethyl ether $\left(\mathrm{C}_{4} \mathrm{H}_{10} \mathrm{O}\right)$ & -7.43301 & 1.78847 & -2.4793 & -3.2811 & 156.86 & 307.59 & 466.74 & 36.50 & 0.282 & 1.3 \\
\hline Decane $\left(\mathrm{C}_{10} \mathrm{H}_{22}\right)$ & -8.60643 & 2.44659 & -4.2925 & -3.9080 & 243.49 & 447.30 & 617.65 & 21.05 & 0.490 & 0.0 \\
\hline Dodecane $\left(\mathrm{C}_{12} \mathrm{H}_{26}\right)$ & -9.08593 & 2.77846 & -5.1985 & -4.1730 & 263.57 & 489.48 & 658.00 & 18.20 & 0.573 & 0.0 \\
\hline
\end{tabular}




\begin{tabular}{|c|c|c|c|c|c|c|c|c|c|c|}
\hline \multirow[b]{2}{*}{ Species } & \multicolumn{4}{|c|}{ Wagner Constants } & \multicolumn{6}{|c|}{ Physical Properties } \\
\hline & $a$ & $b$ & $c$ & $d$ & $T_{f}(\mathrm{~K})$ & $T_{b}(\mathrm{~K})$ & $T_{c}(\mathrm{~K})$ & $P_{c}($ bar $)$ & $\omega$ & $\begin{array}{c}\mu \\
\text { (Debye) }\end{array}$ \\
\hline Eicosane $\left(\mathrm{C}_{20} \mathrm{H}_{42}\right)$ & -10.97958 & 4.25588 & -8.9573 & -5.0430 & 309.95 & 616.84 & 769.00 & 11.60 & 0.891 & \\
\hline Ethane $\left(\mathrm{C}_{2} \mathrm{H}_{6}\right)$ & -6.47500 & 1.41071 & -1.1440 & -1.8590 & 90.35 & 184.55 & 305.33 & 48.71 & 0.099 & 0.0 \\
\hline Heptadecane $\left(\mathrm{C}_{17} \mathrm{H}_{36}\right)$ & -10.23600 & 3.54177 & -7.1898 & -5.0000 & 295.13 & 574.56 & 735.00 & 13.70 & 0.772 & 0.0 \\
\hline n-Heptane $\left(\mathrm{C}_{7} \mathrm{H}_{16}\right)$ & -7.77404 & 1.85614 & -2.8298 & -3.5070 & 182.59 & 371.57 & 540.15 & 27.35 & 0.350 & 0.0 \\
\hline Hexadecane $\left(\mathrm{C}_{16} \mathrm{H}_{34}\right)$ & -10.03664 & 3.41426 & -6.8627 & -4.8630 & 291.32 & 559.98 & 722.00 & 14.35 & 0.737 & 0.0 \\
\hline n-Hexane $\left(\mathrm{C}_{6} \mathrm{H}_{14}\right)$ & -7.53998 & 1.83759 & -2.5438 & -3.1630 & 177.84 & 341.88 & 507.90 & 30.35 & 0.299 & 0.0 \\
\hline Methane $\left(\mathrm{CH}_{4}\right)$ & -6.02242 & 1.26652 & -0.5707 & -1.366 & 90.69 & 111.66 & 190.55 & 45.99 & 0.011 & 0.0 \\
\hline Nonadecane $\left(\mathrm{C}_{19} \mathrm{H}_{40}\right)$ & -10.68217 & 3.98054 & -8.3030 & -4.9950 & 305.25 & 602.34 & 758.00 & 12.30 & 0.844 & \\
\hline Nonane $\left(\mathrm{C}_{9} \mathrm{H}_{20}\right)$ & -8.32886 & 2.25707 & -3.8257 & -3.7320 & 219.66 & 423.97 & 594.90 & 22.90 & 0.443 & 0.0 \\
\hline Octadecane $\left(\mathrm{C}_{18} \mathrm{H}_{38}\right)$ & -10.47230 & 3.69655 & -7.5779 & -5.1090 & 301.32 & 588.30 & 746.00 & 13.00 & 0.812 & 0.0 \\
\hline Octane $\left(\mathrm{C}_{8} \mathrm{H}_{18}\right)$ & -8.04937 & 2.03865 & -3.3120 & -3.6480 & 216.39 & 398.82 & 568.95 & 24.90 & 0.397 & 0.0 \\
\hline Pentadecane $\left(\mathrm{C}_{15} \mathrm{H}_{32}\right)$ & -9.80239 & 3.29217 & -6.5317 & -4.5840 & 283.08 & 543.83 & 708.00 & 15.15 & 0.696 & 0.0 \\
\hline n-Pentane $\left(\mathrm{C}_{5} \mathrm{H}_{12}\right)$ & -7.30698 & 1.75845 & -2.1629 & -2.9130 & 143.43 & 309.22 & 469.80 & 33.75 & 0.251 & 0.0 \\
\hline Propane $\left(\mathrm{C}_{3} \mathrm{H}_{8}\right)$ & -6.76368 & 1.55481 & -1.5872 & -2.024 & 85.47 & 231.02 & 369.83 & 42.48 & 0.152 & 0.0 \\
\hline $\mathrm{R} 152 \mathrm{a}\left(\mathrm{C}_{2} \mathrm{H}_{4} \mathrm{~F}_{2}\right)$ & -7.433439 & 1.755544 & -2.169951 & -2.774693 & 156.15 & 249.10 & 386.41 & 45.17 & 0.275 & 2.3 \\
\hline n-Tetradecane $\left(\mathrm{C}_{14} \mathrm{H}_{30}\right)$ & -9.54470 & 3.06637 & -6.0070 & -4.5300 & 279.01 & 526.76 & 693.00 & 16.10 & 0.654 & 0.0 \\
\hline Tridecane $\left(\mathrm{C}_{13} \mathrm{H}_{28}\right)$ & -9.32959 & 2.89925 & -5.5550 & -4.4700 & 267.76 & 508.63 & 676.00 & 17.10 & 0.618 & 0.0 \\
\hline Undecane $\left(\mathrm{C}_{11} \mathrm{H}_{24}\right)$ & -8.85076 & 2.60205 & -4.7305 & -4.0810 & 247.57 & 469.08 & 638.85 & 19.55 & 0.533 & 0.0 \\
\hline Argon (Ar) & -5.926538 & 1.208266 & -0.509886 & -1.590893 & 83.80 & 87.27 & 150.69 & 48.63 & -0.002 & \\
\hline Nitrogen $\left(\mathrm{N}_{2}\right)$ & -6.11102 & 1.2189 & -0.69366 & -1.89893 & 63.15 & 77.35 & 126.20 & 34.00 & 0.037 & 0.0 \\
\hline Ammonia $\left(\mathrm{NH}_{3}\right)$ & -7.28322 & 1.5716 & -1.85672 & -2.39312 & 195.41 & 239.82 & 405.50 & 113.53 & 0.256 & 1.5 \\
\hline Water $\left(\mathrm{H}_{2} \mathrm{O}\right)$ & -7.861942 & 1.879246 & -2.266807 & -2.128615 & 273.15 & 373.14 & 647.10 & 220.64 & 0.344 & 1.8 \\
\hline Helium (He normal) & -4.265233 & 1.571259 & 0.479795 & 0.751271 & 2.15 & 4.24 & 5.20 & 2.27 & -0.382 & \\
\hline Hydrogen $\left(\mathrm{H}_{2}\right.$ normal $)$ & -4.902616 & 1.065004 & 0.737305 & 0.053125 & 13.56 & 20.37 & 33.15 & 12.96 & -0.219 & \\
\hline
\end{tabular}

All Wagner constants are from Ref. [5], except for helium, hydrogen, argon, water, and R152a, which are from Ref. [17]. All dipole moments and normal fusion points are from Ref. [5]. All critical points are from Ref. [5], except for helium, hydrogen, argon, water, and R152a, which are from NIST [21]. The normal boiling point temperature $\left(T_{b}\right)$ and the acentric factor $(\omega)$ are calculated from the entire-curve Wagner constants; thus, the values of the anchor points of the Riedel and Ambrose-Walton equations are consistent for each species with the corresponding entire-curve Wagner constants.

Table 2. Hypothesized Performance Comparison of Modified Correlations vs. Standard Counterparts.

\begin{tabular}{|c|c|c|c|c|}
\hline \multirow{2}{*}{$T_{r, b}$ vs. 0.7} & \multicolumn{2}{|c|}{$T_{r}<T_{r, b}$} & \multicolumn{2}{|c|}{$T_{r}>T_{r, b}$} \\
\hline & $R$ vs. $R(\omega)$ & A-W vs. A-W $\left(T_{b}\right)$ & $R$ vs. $R(\omega)$ & $\mathrm{A}-\mathrm{W}$ vs. A-W $\left(T_{b}\right)$ \\
\hline$\overline{T_{r, b}>0.7}$ & $\mathrm{R}(\omega)$ & ----- & ----- & $\mathrm{A}-\mathrm{W}\left(T_{b}\right)$ \\
\hline$T_{r, b}<0.7$ & --- & $\mathrm{A}-\mathrm{W}\left(T_{b}\right)$ & $\mathrm{R}(\omega)$ & ---- \\
\hline \multicolumn{5}{|c|}{$\begin{array}{l}\mathrm{R}=\text { standard Riedel } \\
\mathrm{R}(\omega)=\text { modified Riedel, Riedel }(\omega) \\
\mathrm{A}-\mathrm{W}=\text { standard Ambrose-Walton } \\
\mathrm{A}-\mathrm{W}\left(T_{b}\right)=\text { modified Ambrose-Walton, Ambrose- }\end{array}$} \\
\hline
\end{tabular}

\subsection{Determining Best Correlation for Below and Above the Normal Boiling Point}

A summary of the comparison of the predictive error of the standard Riedel and Ambrose-Walton equations and their modified forms at the normal fusion point is shown in Table 3. The absolute percent error (A\%Err), given by $100 * \mid\left(P_{v, r}\right.$ reference $-P_{v, r}$ predicted) $/ P_{v, r}$ reference / where the reference value is represented by the entire-curve Wagner analytic. The average errors for each substance subset is shown for each correlation in the table. The correlation with the average least error when the standard Riedel and Ambrose-Walton go head-to-head is indicated, and that with the least average error of all four correlations is also indicated ("All Four" column).
Comparing all four correlations to each other, Table 3 shows that $\mathrm{R}(\omega)$ results in lowest error for organic acids, Riedel for alcohols, Ambrose-Walton for "Others" with $T_{r, b}$ $>0.7$, and A-W( $\left.T_{b}\right)$ for "Others" with $T_{r, b}<0.7$. The "All Four" entries that match the hypotheses are in italic font, those contradicting a hypothesis are shaded in gray, and the cells with normal font and no shading are cases for which there are no expectations. It is seen that when averaged over each substance type, the modified Riedel does meet expectations for organic acids - Riedel outperformed Ambrose-Walton, and $\mathrm{R}(\omega)$ has the least error of all correlations. However, $\mathrm{R}(\omega)$ does not meet expectations for alcohols with $T_{r, b}>0.7$; it does not have the least error of all four correlations despite Riedel outperforming AmbroseWalton. A-W $\left(T_{b}\right)$ met expectations because it 
Table 3. Summary of Comparison of Standard and Modified Riedel and Ambrose-Walton Equations: A\%Err @ $T_{r, f}$ Relative to Entire-Curve Wagner Analytics.

\begin{tabular}{|c|c|c|c|c|c|c|}
\hline & \multicolumn{4}{|c|}{ Average A\%Err } & \multicolumn{2}{|c|}{ Least A\%Err } \\
\hline & $\mathrm{R}$ & $\mathrm{R}(\omega)$ & A-W & $\mathrm{A}-\mathrm{W}\left(T_{b}\right)$ & R vs. A-W & All Four ${ }^{1}$ \\
\hline \multicolumn{7}{|c|}{ Acids (9) } \\
\hline$T_{r, b}>0.7(6)$ & 41.02 & 23.71 & 107.57 & 152.19 & $\mathrm{R}$ & $\mathrm{R}(\omega)$ \\
\hline$T_{r, b}<0.7(3)$ & 12.28 & 5.33 & 27.29 & 23.25 & $\mathrm{R}$ & $\mathrm{R}(\omega)$ \\
\hline \multicolumn{7}{|c|}{ Alcohols (22) } \\
\hline$T_{r, b}>0.7(13)$ & 289.21 & 572.54 & $1,827.82$ & $1,989.41$ & $\mathrm{R}$ & $\mathrm{R}$ \\
\hline$T_{r, b}<0.7(9)$ & 71.99 & 310.04 & 680.22 & 639.65 & $\mathrm{R}$ & $\mathrm{R}$ \\
\hline \multicolumn{7}{|c|}{ "Others" (41) } \\
\hline$T_{r, b}>0.7(14)$ & 28.81 & 44.91 & 3.59 & 4.03 & A-W & A-W \\
\hline$T_{r, b}<0.7(27)$ & 18.59 & 44.98 & 8.22 & 7.17 & A-W & A-W(Tb) \\
\hline Normal (21) & 17.99 & 49.78 & 5.17 & 4.85 & A-W & A-W(Tb) \\
\hline Polar (6) & 20.69 & 28.18 & 18.93 & 15.13 & A-W & A-W(Tb) \\
\hline $\begin{array}{l}\mathrm{R}=\text { Riedel; } \mathrm{R}(\omega)=\mathrm{R} \\
{ }^{1} \text { Those that match th } \\
\text { and cells with normal }\end{array}$ & $\begin{array}{l}\operatorname{del}(\omega) \text {; A } \\
\text { hypothes } \\
\text { ont and } n\end{array}$ & $\begin{array}{l}\mathrm{V}=\mathrm{Amb} \\
\text { are in ita } \\
\text { hading a }\end{array}$ & $\begin{array}{l}\text { e-Walton; } \\
\text { font, thos } \\
\text { cases for w }\end{array}$ & $\begin{array}{l}\mathrm{A}-\mathrm{W}\left(T_{b}\right) \\
\text { ontradictin } \\
\mathrm{h} \text { there are }\end{array}$ & $\begin{array}{l}\text { mbrose-Walt } \\
\text { hypothesis ar } \\
\text { expectations. }\end{array}$ & aded in gray, \\
\hline
\end{tabular}

both has the least error for all species where $T_{r, b}<0.7$ and the standard Ambrose-Walton performs better than Riedel.

Organic acids with $T_{r, b}<0.7$ is one cell having no expectations, for which $\mathrm{R}(\omega)$ has the least average predictive error. Restricting the comparison to just modified vs. standard forms, one can see that the R( $\omega)$ correlation has better predictive power than the standard Riedel only for organic acids. The A-W $\left(T_{b}\right)$, however, is superior to its standard counterpart for all substance types with $T_{r, b}<0.7$.

Table 4 summarizes the results for the equally-weighted average absolute percent errors for the parts of the two-phase VLE curve, both below and above the normal boiling point. The average for a subset shown in Table 4 is not the average of all the corresponding species' averages in that subset, but rather it is the sum of the individual $\mathrm{A} \%$ Err values for the VLE points of all species in that subset divided by the number of total points in the subset. This is done because the number of points above and below the normal boiling point varies for each species given that the value of the normal boiling point temperature varies. The normal boiling point itself is not included in either of the two temperature segments.

The correlation that has the least predictive error for each substance subset is shown in the "All Four" column, segregated further by location of the normal boiling point. The normal fusion point generally has the largest error along the entire two-phase curve, thus, it tends to dominate when included in averages of equally-weighted points. This evident by the fact that the "All Four" entries for the $T_{r}<T_{r, b}$ segment are the same as those for the single normal fusion point shown in Table 3, except that the standard Riedel is superior to the standard Ambrose-Walton in their head-tohead competition for the 6 polar "Others" when $T_{r, b}<0.7$. There are no polar "Others" with $T_{r, b}>0.7$ for comparison in our sample pool of 72 species.

Only one subset of species has results contrary to the hypothesized relative performance - alcohols with $T_{r, b}>0.7$, which cells are shaded in gray; $R(\omega)$ is expected to be best performer of "All Four" below the normal boiling point, and A-W $\left(T_{b}\right)$ above. The "All Four" entries of the other subsets are consistent with the expectations and are shown in italic font. The subsets for which there were no expectations are shown in normal font without shading. Note that $\mathrm{R}(\omega)$ has the least predictive error for the $T_{r}>T_{r, b}$ segment for all substance subsets except "Others" with $T_{r, b}>0.7$.

Because the error at low temperatures tend to dominate the entire-curve statistics when points are equally weighted, the entries in the "All Four" column for the $T_{r}<T_{r, b}$ segment also apply to the entire curve, i.e., the correlation that has the least error for a species subset at the low-temperature end of the curve ends up also having the least error in aggregate over the entire curve when the four correlations are compared head-to-head. The $F_{w, j}$ function, however, will be shown to provide a method to combine the best correlations below and above the normal boiling point to reduce the average error over the entire two-phase curve.

The results shown in Table 4 suggest ways to combine the correlations via the $F_{w, j}$ function:

1) $R(\omega)$ appears to be best for the entire two-phase coexistence curve for organic acids, and yet there is still significant predictive error, so four $\mathrm{R}(\omega)$ points are used to estimate Wagner constants to assess whether or not the $F_{w, j}$ function can improve upon the $\mathrm{R}(\omega)$ correlation. The Wagner constants estimated by Eq. (6) from such combinations are denoted by $F_{w, j}[R(\omega)]$.

2) It is hypothesized that the predictive error over the entire curve for alcohols can be decreased by combining Riedel points below the normal boiling point with $R(\omega)$ points above the normal boiling point, and the Wagner constants estimated by Eq. (6) from such combinations are denoted by $F_{w, j}[R \mid R(\omega)]$.

3) The standard Ambrose-Walton cannot be outperformed in aggregate for "Others" with $T_{r, b}>0.7$ either below or above the normal boiling point, and the average errors are sufficiently small such that only minimal benefit could potentially be achieved by $F_{w, j}$ functions based on vapor pressure predictions of the Ambrose-Walton. Thus, this subset of species is not targeted by $F_{w, j}$ functions.

4) And lastly, it is hypothesized that error can be reduced for "Others" with $T_{r, b}<0.7$ by using A-W $\left(T_{b}\right)$ points below the normal boiling point and $\mathrm{R}(\omega)$ points above, and the 
equations for the estimated Wagner constants for such combinations are denoted by $F_{w, j}\left[A-W\left(T_{b}\right) \mid R(\omega)\right]$.

Three different point distributions are used. Because of the general characteristics of the $\mathrm{S}$ shape of the $\log P_{v}$ vs. $1 / T$ curve, we try to capture the best predictions from both below and above the normal boiling point. A reduced temperature $\geq 0.9$ is included to try to incorporate the region around the upper inflection point. The vapor pressure at the reduced temperature of 0.6 has been shown to significantly increase the prediction of VLE and other thermodynamic properties for polar fluids when combined with the acentric factor [22][24]. The predictive error of the four correlations involved generally increases as the saturation temperature decreases, with the error increasing significantly at reduced temperatures lower than 0.6. Although the predictions of the four correlations being used are not what would be considered good precision compared to actual data, the four saturation points at the reduced temperatures of $0.6,0.7,0.8$, and 0.9 are initially chosen for use in the $F_{w, j}$ function because it represents an interval of decent width and located in the part of the VLE curve with the least predictive error by the four correlations. The superscript "(1)" is used to indicate this point distribution for $F_{w, j}$ functions comprised of points from two correlations.

However, that set of points can exclude the standard Riedel and A-W $\left(T_{b}\right)$ being used if the normal boiling point's reduced temperature is less than 0.6. Consequently, the set of reduced temperatures $0.3,0.6,0.7$, and 0.95 are used to extend the interval below any reasonable normal boiling point and extend the interval further upward into the area of the upper inflection point. The reduced temperatures of 0.6 and 0.7 are chosen to help establish the middle of the VLE curve with accurate data since standard Riedel and A-W $\left(T_{b}\right)$ have the normal boiling point as an anchor and $\mathrm{R}(\omega)$ has the acentric factor as an additional anchor. The superscript "(2)" is used to indicate this point distribution
The third set of points include the reduced temperatures $0.2, T_{r, b}, 0.7$, and 0.95 . The low end of the interval is lowered to 0.2 to account for those species where the below- $T_{b}$ correlation is accurate, and the normal boiling point is used to enforce zero error at this known point, thereby providing good representation for the below- $T_{b}$ segment of the twophase VLE curve for such species. The normal boiling point pressure, 1.01325 bars, is used for $T_{r, b}$. The superscript "(3)" is used to indicate this point distribution.

The temperature of the triple point (or normal fusion point) is not always known, thus, the lowest reduced temperature of the four-point distributions used is fixed rather than being tied to a species-dependent lower bound. Additional implementation specifics are given below for some $F_{w, j}$ functions.

\subsection{1 $\boldsymbol{F}_{w, j}[R(\omega)]$}

$\mathrm{R}(\omega)$ predictions at the four reduced temperatures of 0.6 , $0.7,0.8$, and 0.9 are used with this function.

\subsection{2 $F_{w, j}^{(3)}[R \mid R(\omega)]$}

The reduced vapor pressure at $T_{r}=0.7$ is from $\mathrm{R}(\omega)$ since it should be accurate at that reduced temperature and be consistent with the Riedel value for $T_{r}=0.2$ given that both correlations have the normal boiling point as an anchor and both have similar functional form.

\subsection{3 $F_{w, j}^{(3)}\left[A-W\left(T_{b}\right) \mid R(\omega)\right]$}

Unlike for $F_{w, j}{ }^{(3)}[R \mid R(\omega)]$, the vapor pressure for $T_{r}=0.7$ in $F_{w, j}{ }^{(3)}\left[A-W\left(T_{b}\right) \mid R(\omega)\right]$ is calculated using the $\mathrm{A}-\mathrm{W}\left(T_{b}\right)$ if 0.7 is below the normal boiling point reduced temperature and $\mathrm{R}(\omega)$ if it is above because $\mathrm{R}(\omega)$ and $\mathrm{A}-\mathrm{W}\left(T_{b}\right)$ do not share a similar functional form.

The point distributions for the three fully-determined cases used in this study to calculate Wagner constants from the seven $F_{w, j}$ functions are summarized in Table 5.

Table 4. Summary of Comparison of Standard and Modified Riedel and Ambrose-Walton Equations: A\%Err for Below \& Above $T_{b}$ Relative to Entire-Curve Wagner Analytics.

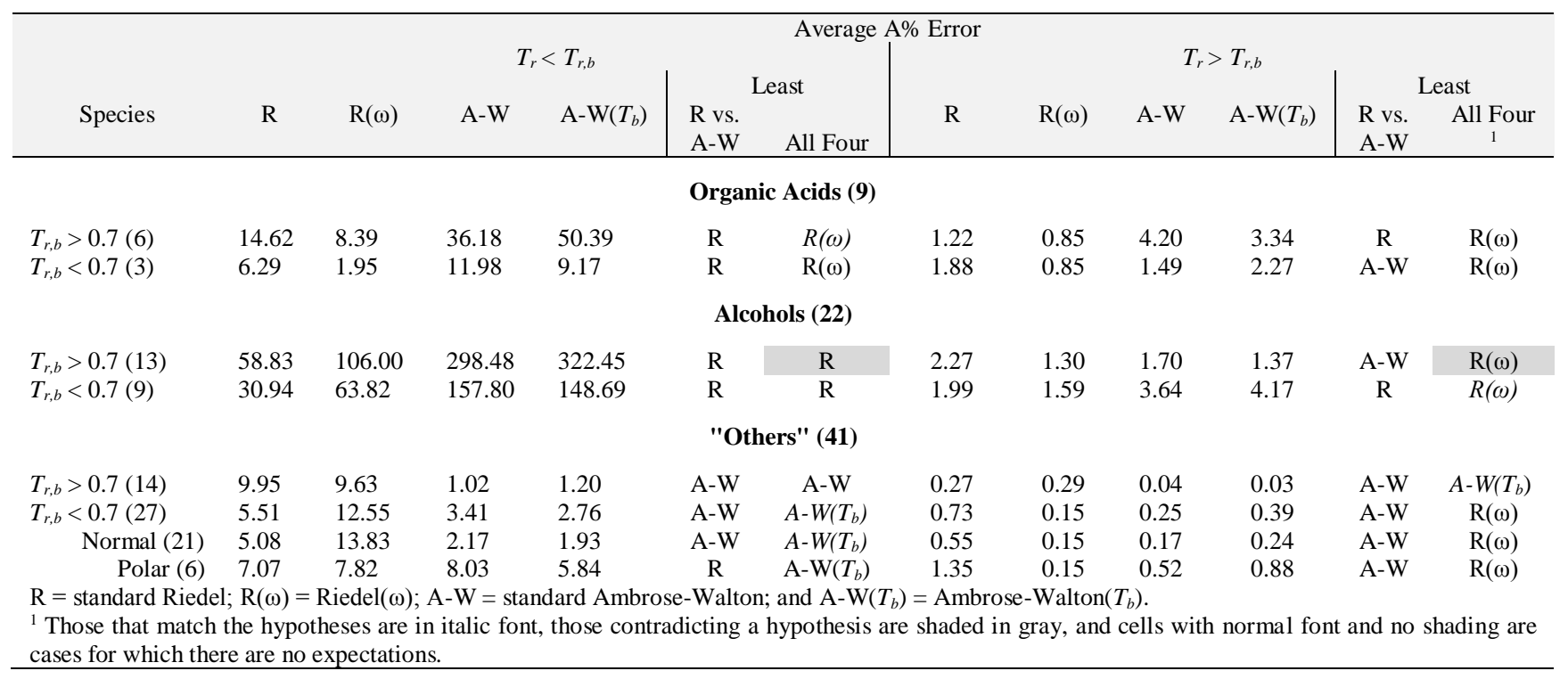


Table 5. Point Distributions and $\eta_{j i}$ Values for the Three Fully-Determined Cases Used.

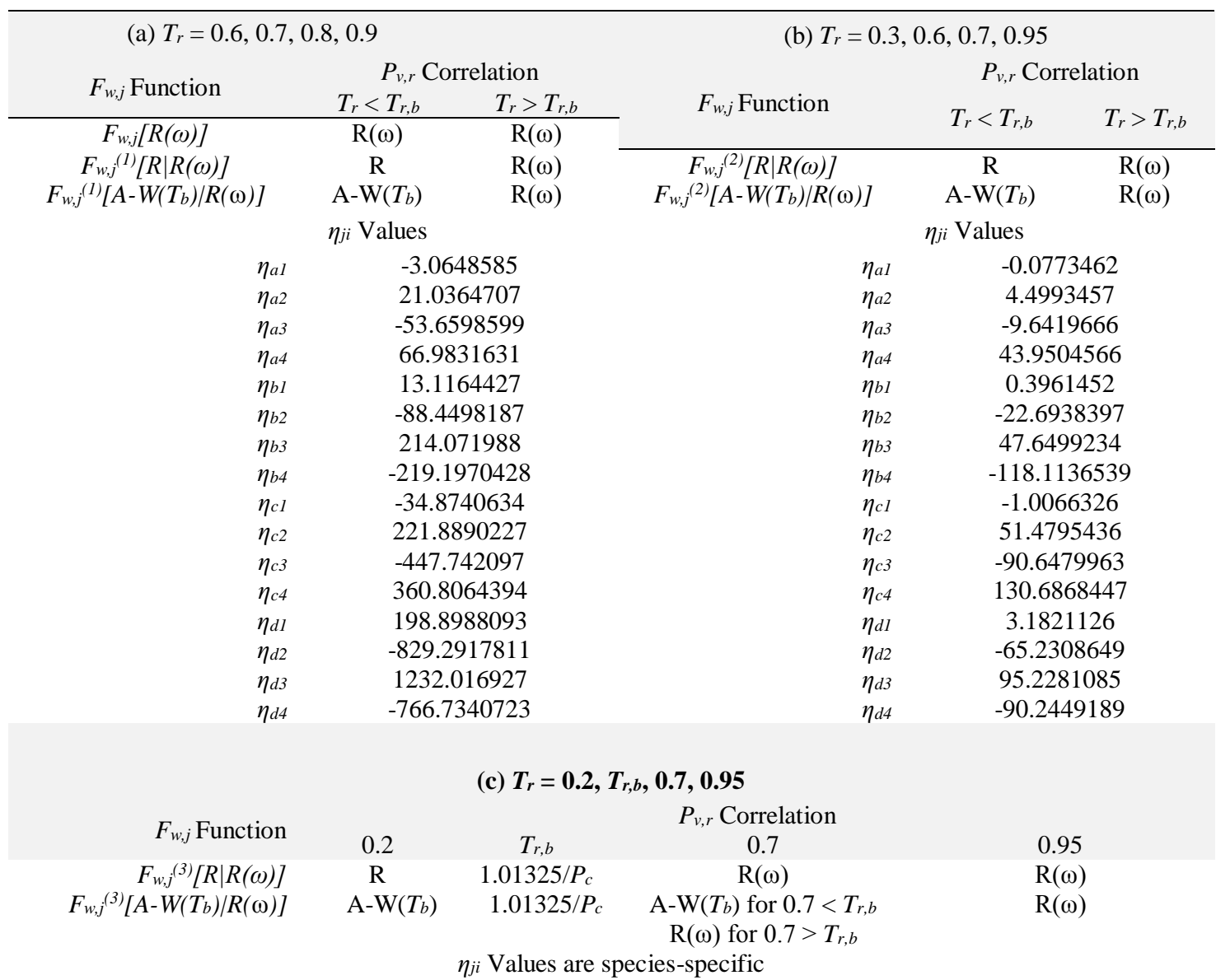

The $\eta_{j i}$ values are shown for cases (a) and (b) because they apply to every species. The $\eta_{j i}$ values for case (c), however, are species-dependent because the normal boiling point is one of the four points. The algebraic equations for the $\eta_{j i}$ parameters, which are dependent only upon the chosen four reduced temperatures, can be found in Ref. [25].

The $\eta_{j i}$ values in Table 5 are shown with seven significant digits right of the decimal point to allow for rounding off of calculated Wagner constants to six digits right of the decimal point. The well-established value of 1.01325 bar is used for the atmospheric pressure for the normal boiling point. Reduced temperatures used as inputs to the calculations are rounded off to five significant digits; however, hanging zeros are dropped from reduced temperature values in the tables. More explanation of the round off used in calculations is provided in Ref. [18].

\section{Results}

When discussing statistics for predicted VLE points, the "All $T_{r} \mathrm{~s}$ " term is used here to indicate the entire two-phase curve, including the normal boiling point. The error at the normal boiling point will not always be zero because not all of the correlations and functions have the normal boiling point as an anchor. The results of the various $F_{w, j}$ functions are summarized in Table 6 for "All $T_{r}$ s." The cell of the function having the least average error for a substance subset is shaded in gray. The type of $F_{w, j}$ function suggested by the correlations that have the least subset errors of the below- $T_{b}$ and above- $T_{b}$ curve segments (see Table 4 ) are also indicated for the species subset. A comparison indicates that some $F_{w, j}$ functions with the least subset error are not consistent with those suggested from the results shown in Table 4 . For example, $F_{w, j}^{(3)}[R \mid R(\omega)]$ has the minimum average error for organic acids rather than $F_{w, j}[R(\omega)]$, and $F_{w, j}[R(\omega)]$ has less error than the $F_{w, j}\left[A-W\left(T_{b}\right) \mid R(\omega)\right]$ functions for polar "Others" with $T_{r, b}<0.7$. On the other hand, consistent with the results in Table 4 are the $F_{w, j}[R \mid R(\omega)]$ functions having the least average error for alcohols and the $F_{w, j}{ }^{(3)}[A$ $\left.W\left(T_{b}\right) \mid R(\omega)\right]$ function having the minimum average error for the twenty-seven "Others" and the twenty-one normal "Others" with $T_{r, b}<0.7$.

Not surprisingly, the $F_{w, j}[R(\omega)]$ and $F_{w, j}[R \mid R(\omega)]$ functions perform best for polar species while $F_{w, j}[A-$ $\left.W\left(T_{b}\right) \mid R(\omega)\right]$ functions have the advantage with normal species. As mentioned previously, no attempt is made to design an $F_{w, j}$ function to outperform the Ambrose-Walton correlation for normal species that have $T_{r, b}>0.7$, but the $F_{w, j}$ function with the least error for this subset is indicated by gray shading in Table 6 for completeness sake.

\subsection{Minimum Possible Entire-Curve Average Error \& Levels of Success}

The minimum possible entire-curve total A\%Err for a given species using the four correlations is the minimum error below the normal boiling point summed with that 
Table 6. Summary of Comparison of Average A\%Err of $F_{w, j}$ Functions for Entire Two-Phase Curve ("All $T_{r} s^{\prime \prime)}$.

Average A\% Err for Entire Curve

Species

$$
F_{w, j}^{(l)} \quad F_{w, j}^{\left({ }^{(2)}\right.} \quad F_{w, j}^{\left({ }^{(3)}\right.} \quad F_{w, j}^{(I)} \quad F_{w, j}^{(2)} \quad F_{w, j}^{(3)}
$$
$F_{w, j}[R(\omega)] \quad[R \mid R(\omega)] \quad[R \mid R(\omega)] \quad[R \mid R(\omega)] \quad\left[A-W\left(T_{b}\right) \mid R(\omega)\right] \quad\left[A-W\left(T_{b}\right) \mid R(\omega)\right] \quad\left[A-W\left(T_{b}\right) \mid R(\omega)\right]$

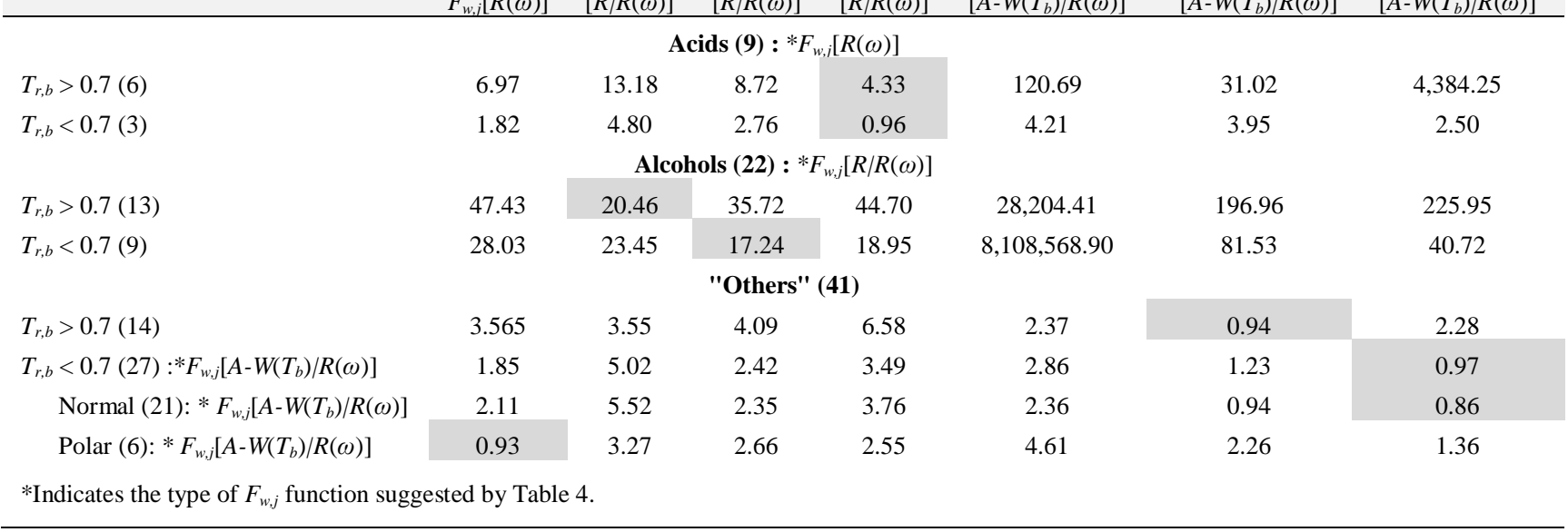

above the normal boiling point. This total "All $T_{r} \mathrm{~s}$ " error is then divided by the number of calculated points plus 1 to arrive at the minimum possible average A\%Err for the entire VLE curve. A detailed description of the concept of minimum possible error and its values for the 72 species studied can be found in Ref. [25].

The minimum possible average error for a species is the best that can be obtained using any combination of the four correlations for the two temperature segments $T_{r}<T_{r, b}$ and $T_{r}$ $>T_{r, b}$. This minimum possible error is the most stringent test because the final Wagner constants chosen for a species would have to blend well the functional form of the best correlation for the $T_{r}<T_{r, b}$ segment with that of the best correlation for the $T_{r}<T_{r, b}$ segment, while accurately predicting the normal boiling point pressure. A less stringent test is having an entire-curve average A\%Err less than all of the four correlations. The least stringent test is having an entire-curve average A\%Err less than just the Riedel and Ambrose-Walton equations. A set of Wagner constants that can predict better than or equal to the standard Riedel and Ambrose-Walton is considered a satisfactory success since those two correlations are the primary tools available and recommended for use when VLE data is sparse. A set of Wagner constants is considered to have superior success if it can also match or beat the additional $\mathrm{R}(\omega)$ and $\mathrm{A}-\mathrm{W}\left(T_{b}\right)$ correlations introduced here since it has been shown that they can perform better than their standard counterparts in many cases. Ultimate success is achieved when the estimated Wagner constants can meet or beat the minimum possible error; a methodology that can on average incorporate the best predictive powers of the four correlations would be remarkable.

\subsection{Comparison to Minimum Possible Entire-Curve Average Error}

Table 7 shows subset least average entire-curve errors for several comparators. The minimum possible average A\%Err is displayed here for reference, given that it is the criterion for Ultimate success. The minimum possible errors indicate a dependency upon relative position of the normal boiling point to the reduced temperature of 0.7 , and the dependency for normal "Others" is reverse of that for organic acids and alcohols. The large average minimum entire-curve errors for alcohols (19.65 and 8.93\%) indicate that none of the four correlations can describe them well, and one might conclude the same for organic acids with $T_{r, b}>0.7$, whose average minimum possible error is $3.93 \%$.

The column to the right of the minimum possible error indicates the least average error one could achieve if one knew a priori which would have the lowest error in head-tohead competition between the standard Riedel and AmbroseWalton correlations for each individual species. Similarly, the next column over displays the least average error one could achieve knowing beforehand which of the four correlations provides the least entire-curve predictive error for each species. The next column shows the least average error possible if one knew which $F_{w, j}$ function was best for each species. The subsequent columns to the right indicate the average errors obtained when the best subset method is applied to every member of the substance subset. The best subset $F_{w, j}$ functions and their corresponding average errors are taken from Table 6, except for "Others" with $T_{r, b}<0.7$, in which case the average is based upon the results of the subset best methods for normal and polar species combined. The subset of normal "Others" species with $T_{r, b}>0.7$ is included for reference as in Table 6, but it is excluded from the following discussion regarding the results of Table 7 since that subset is not targeted.

Looking at the averages of the species-specific least errors, the $F_{w, j}$ functions produce less error than do the four correlations, which in turn result in less error than when limited to only the standard Riedel and Ambrose-Walton. Interestingly, the $F_{w, j}$ functions result in less average error than the minimum possible error for every species subset, whereas, the four correlations do not. In other words, four vapor pressures predicted by two of the four correlations can be used via the $F_{w, j}$ functions to result in less error than the individual correlations themselves produce over the entire curve and also less than when the entire-curve error is minimized for a species by splitting the VLE curve at the normal boiling point and using the best correlation for each temperature segment. The fact that a fully-determined formulation such as the $F_{w, j}$ function based on vapor pressure predictions can outperform the source correlations is a testament to the robustness of the Wagner equation and indicates that the fully-determined case can improve VLE 
Table 7. Summary Comparison of Entire-Curve Average Absolute Percent Error.

\begin{tabular}{|c|c|c|c|c|c|c|c|c|c|}
\hline \multirow{2}{*}{$T_{r, b}$ vs. 0.7} & \multicolumn{4}{|c|}{$\begin{array}{l}\text { Using Species-Specific Least Average } \\
\text { A\%Err }\end{array}$} & \multicolumn{5}{|c|}{ Average A\%Err Using Subset Best Method } \\
\hline & $\begin{array}{l}\text { Minimum } \\
\text { Possible }\end{array}$ & $\begin{array}{l}\text { R vs. A- } \\
\text { W }\end{array}$ & $\begin{array}{c}\text { All } \\
\text { Four }\end{array}$ & $F_{W, j}$ & \multicolumn{2}{|c|}{ Correlation } & & $F_{W, j}$ & $\begin{array}{c}F_{W, j} \\
\text { Success }\end{array}$ \\
\hline \multicolumn{10}{|c|}{ Acids (9) } \\
\hline$T_{r, b}>0.7(6)$ & 3.93 & 7.84 & 4.09 & 3.12 & 5.07 & $\mathrm{R}(\omega)$ & 4.33 & $F_{w, j}^{(3)}[R \mid R(\omega)]$ & $\mathrm{S}$ \\
\hline$T_{r, b}<0.7$ & 0.99 & 3.18 & 1.26 & 0.66 & 1.26 & $\mathrm{R}(\omega)$ & 0.96 & $F_{w, j}^{(3)}[R \mid R(\omega)]$ & $\mathrm{U}$ \\
\hline \multicolumn{10}{|c|}{ Alcohols (22) } \\
\hline$T_{r, b}>0.7(13)$ & 19.65 & 21.13 & 20.12 & 8.95 & 35.68 & $\mathrm{R}$ & 20.46 & $F_{w, j}^{(l)}[R \mid R(\omega)]$ & $\mathrm{S}$ \\
\hline$T_{r, b}<0.7(9)$ & 8.93 & 13.37 & 9.22 & 7.80 & 17.59 & $\mathrm{R}$ & 17.24 & $F_{w, j}^{(2)}[R \mid R(\omega)]$ & $<\mathrm{S}$ \\
\hline \multicolumn{10}{|c|}{ "Others" (41) } \\
\hline$T_{r, b}>0.7(14)$ & 0.49 & 0.66 & 0.50 & 0.73 & 0.66 & A-W & 0.94 & $F_{w, j}^{(2)}\left[A-W\left(T_{b}\right) \mid R(\omega)\right]$ & $<\mathrm{S}$ \\
\hline$T_{r, b}<0.7(27)$ & 1.07 & 1.50 & 1.13 & 0.78 & 1.44 & $\mathrm{~A}-\mathrm{W}\left(T_{b}\right)$ & 0.87 & See breakdown below & $\mathrm{U}$ \\
\hline Normal (21) & 0.81 & 1.02 & 0.84 & 0.75 & 1.00 & $\mathrm{~A}-\mathrm{W}\left(T_{b}\right)$ & 0.86 & $F_{w, j}^{(3)}\left[A-W\left(T_{b}\right) \mid R(\omega)\right]$ & $\mathrm{S}$ \\
\hline Polar (6) & 1.99 & 3.18 & 2.12 & 0.91 & 2.98 & $\mathrm{~A}-\mathrm{W}\left(T_{b}\right)$ & 0.93 & $F_{w, j}[R(\omega)]$ & $\mathrm{U}$ \\
\hline \multicolumn{10}{|c|}{$\begin{array}{l}\text { Legend for Success Levels: " }<\mathrm{S} \text { " indicates less than Satisfactory (average error of subset best } F_{w, j} \text { function }>\text { species-specific subset average } \\
\text { error of either standard Riedel or Ambrose-Walton). "S" indicates Satisfactory (average error of subset best } F_{w, j} \text { function } \leq \text { species-specific } \\
\text { subset average error of both standard Riedel and Ambrose-Walton). "U" indicates Ultimate (average error of subset best } F_{w, j} \text { function } \leq \text { species- } \\
\text { specific subset average minimum possible error). Note that achieving Superior success (average error of subset best } F_{w, j} \text { function } \leq \text { species- } \\
\text { specific subset average error for all four correlations) without achieving Ultimate success does not occur. }\end{array}$} \\
\hline
\end{tabular}

predictive error if used wisely. These results provide strong support for the earlier preliminary assessment [18] that Wagner constants estimated from predictions can perform better than the source correlations.

As shown in Tables 4 and 6, however, no single correlation or $F_{w, j}$ function results in the least error for all species, let alone for each member of a subset of species. Indeed, individual correlations and $F_{w, j}$ functions perform very poorly for some species. Thus, benefit to the practitioner can result only if subset best methods can perform well relative to the minimum possible error. This comparison is made with the "Average Using Subset Best Method" columns. In every case the subset best $F_{w, j}$ function has less average predictive error than the subset best correlation. The last column of Table 7 shows the success level of the subset best $F_{w, j}$ function. The success level is less than satisfactory for only alcohols with $T_{r, b}<0.7$. Although $F_{w, j}^{(2)}[R \mid R(\omega)]$, the best $F_{w, j}$ function for this subset of alcohols (17.24\%), does have slightly less error than the subset best correlation, standard Riedel (17.59\%), it is greater than the error obtainable if one knows a priori for each species the best of all four correlations $(9.22 \%)$ or the best between just the standard Riedel and Ambrose-Walton $(13.37 \%)$. The subset best $F_{w, j}$ functions achieve either satisfactory or ultimate success for the other substance subsets. One can see that there is no "Superior" entry in the "Success" column - if the subset best $F_{w, j}$ function can outperform the species-specific "All Four" average error, then it also ends up beating the minimum possible error and achieving Ultimate success.

Table 7 overall shows that subset best $F_{w, j}$ functions have better predictive power than subset best correlations, except for "Others" with $T_{r, b}>0.7$ (all normal species except for helium), which is not a targeted species subset. The subset best $F_{w, j}$ functions achieve ultimate success in aggregate for 30 of the 72 species. All six $F_{w, j}$ functions shown in Table 7 pass the qualitative Waring fit-of-form test for all species in their corresponding substance subset - the constants $b$ and $c$ had opposite signs for all species, except helium and hydrogen, whose normal boiling points are below $50 \mathrm{~K}$.

The data shown in Table 7 for alcohols is especially interesting. First, the species-specific $F_{w, j}$ functions produce much less error than the minimum possible $(8.95 \%$ vs. $19.65 \%$ and $7.80 \%$ vs. $8.93 \%$ ), indicating that improvement in predictive error can be achieved by combining predicted VLE from two of the four correlations for individual species; however, neither a single correlation for either the belowand above- $T_{b}$ segments nor $F_{w, j}$ function for the entire VLE curve describe the alcohol subset well enough for one to be confident of which to use for a given alcohol a priori.

Second, for alcohols with $T_{r, b}<0.7$, the subset best $F_{w, j}$ function is only slightly better than the subset best correlation, 17.24 and $17.59 \%$, respectively. Consequently, one may decide to use the Riedel correlation instead of the more algebraically cumbersome $F_{w, j}^{(2)}[R \mid R(\omega)]$ function for alcohols with $T_{r, b}<0.7$. The nine alcohols in this subset share three different species-specific correlation winners at the normal fusion point and six different $F_{w, j}$ function winners for the entire two-phase curve. The lack of a correlation that can consistently describe the VLE below the normal boiling point accurately for these alcohols results in the large entire-curve minimum possible error of $8.93 \%$ and subsequently prohibits a single correlation or $F_{w, j}$ function from being successful. Consequently, the results do not strongly recommend any combination of the underlying four correlations used in this work to predict VLE for this species subset. 
Third, although the subset best function $F_{w, j}^{(l)}[R \mid R(\omega)]$ is significantly better than the subset best correlation Riedel for alcohols with $T_{r, b}>0.7$ and approaches closely the minimum possible error of $19.65 \%$, it unfortunately still has a large error of $20.46 \%$. The results for individual species indicate that the $\mathrm{A}-\mathrm{W}\left(T_{b}\right)$ correlation and the $F_{w, j}{ }^{(1)}\left[A-W\left(T_{b}\right) \mid R(\omega)\right]$ function perform significantly better than the subset best methods for the four alcohols with $T_{r, b}>0.76$ (e.g., 1eicosanol, 1-octadecanol, 1-heptadecanol, and 1hexadecanol). The actual appropriate reduced temperature threshold may be lower, but the limited sample pool in our analysis suggests a value near 0.76 .

Table 8 shows the average errors when the alcohols $T_{r, b}$ $>0.7$ subset is divided further to account for the 0.76 threshold. The best $F_{w, j}$ function for the two new alcohol subsets achieve Ultimate success while only Satisfactory success is achieved by the aggregate $T_{r, b}>0.7$ segment best function $F_{w, j}^{(1)}[R \mid R(\omega)]$.

Even though the $F_{w, j}\left[A-W\left(T_{b}\right) \mid R(\omega)\right]$ function has lower error than the A-W $\left(T_{b}\right)$ correlation for alcohols with $T_{r, b}>$ 0.76 , the $F_{w, j}\left[A-W\left(T_{b}\right) \mid R(\omega)\right]$ function can exhibit disproportionate errors for other alcohols because of the poor predictive behavior of Ambrose-Walton at low reduced temperatures for alcohols in general, which poor predictive power is compounded when its low-temperature prediction is used as one of the four points. Consequently, the A-W $\left(T_{b}\right)$ correlation is recommended for alcohols with $T_{r, b}>0.76$ because it doesn't induce this potential compound effect. One can see from Table 8 that some error reduction is lost by using $\mathrm{A}-\mathrm{W}\left(T_{b}\right)$ instead of $F_{w, j}{ }^{(1)}\left[A-W\left(T_{b}\right) \mid R(\omega)\right]$ (average error of $15.86 \%$ compared to $12.95 \%$ ) for this species subset, but the goal of minimizing maximum error and not just minimizing average error drives this recommendation.

Table 9 summarizes the average incremental errors for the recommended correlations and $F_{w, j}$ functions, relative to the minimum possible entire-curve error. By definition, every negative incremental error for a subset corresponds to an aggregate ultimate success level. The average entirecurve error reduction of $13.22 \%$ for some alcohols and the $1.06 \%$ reduction for polar "Others" are strong evidence of the robustness of the Wagner equation and the utility of the fully-determined method employing the $F_{w, j}$ function for substance subsets.

\subsection{Impact of Using Predictions Below the Known Anchor Points: Extrapolation Burden}

A normalized extrapolation burden for a predictive method was defined previously [18] as $\Delta \log P_{v, r} / \Delta T_{r}$, where $\Delta T_{r}$ represents the difference between the reduced normal fusion point and the reduced temperature of the lowest known vapor pressure (the lowest known anchor point) used by the predictive method, and $\Delta \log P_{v, r}$ represents the difference between the base 10 logarithm of the "best" values of the reduced vapor pressures at these two temperatures. The dimensionless numerator $\Delta \log P_{v, r}$ in this paper is referred to separately as the non-normalized extrapolation burden.

Except at low values of extrapolation burden, a correlation's fixed value or rule for the lower anchor point generally results in larger values of non-normalized burdens having correspondingly larger values of normalized burdens.

Table 8. Summary Comparison of Entire-Curve Average Absolute Percent Error - Alcohol Subsets for $T_{r, b}>0.7$.

\begin{tabular}{|c|c|c|c|c|c|c|c|c|c|}
\hline \multirow{3}{*}{$\begin{array}{l}\boldsymbol{T}_{\boldsymbol{r}, \boldsymbol{b}} \text { vs. } \mathbf{0 . 7} \\
T_{r, b}>0.7(13)\end{array}$} & \multicolumn{4}{|c|}{ Using Species-Specific Least Error } & \multicolumn{5}{|c|}{ Using Best Subset Method } \\
\hline & $\begin{array}{c}\text { Minimum } \\
\text { Possible }\end{array}$ & $\begin{array}{c}\text { R vs. A- } \\
\text { W }\end{array}$ & All Four & $\boldsymbol{F}_{w, j}$ & \multicolumn{2}{|c|}{ Correlation } & \multicolumn{2}{|r|}{$\boldsymbol{F}_{w, j}$} & $\begin{array}{c}\boldsymbol{F}_{w, j} \\
\text { Success }\end{array}$ \\
\hline & 19.65 & 21.13 & 20.12 & 8.95 & 35.68 & $\mathrm{R}$ & 20.46 & $F_{w, j}^{(I)}[R \mid R(\omega)]$ & $\mathrm{S}$ \\
\hline$T_{r, b}>0.76(4)$ & 15.85 & 18.28 & 15.86 & 12.95 & 15.86 & $\mathrm{~A}-\mathrm{W}\left(T_{b}\right)$ & 12.95 & $F_{w, j}^{(l)}\left[A-W\left(T_{b}\right) \mid R(\omega)\right]$ & $\mathrm{U}$ \\
\hline $0.7<T_{r, b}<0.76$ & 21.33 & 22.39 & 22.00 & 7.19 & 22.39 & $\mathrm{R}$ & 8.11 & $F_{w, j}^{(l)}[R \mid R(\omega)]$ & $\mathrm{U}$ \\
\hline
\end{tabular}

Table 9. Summary of Entire-Curve Incremental Error for Recommended Predictive Methods.

\begin{tabular}{|c|c|c|c|c|}
\hline \multirow[b]{2}{*}{$T_{r, b}$ vs. 0.7} & \multirow{2}{*}{$\begin{array}{c}\text { Minimum Possible } \\
\text { Average A\%Err }\end{array}$} & \multicolumn{3}{|c|}{ Subset Predictive Recommendation } \\
\hline & & $\begin{array}{c}\text { Average } \\
\text { A\%Err }\end{array}$ & Method & Incremental Error \\
\hline \multicolumn{5}{|c|}{ Organic Acids (9) } \\
\hline $\begin{array}{l}T_{r, b}>0.7 \text { (6) } \\
T_{r, b}<0.7 \text { (3) }\end{array}$ & $\begin{array}{l}3.93 \\
0.99\end{array}$ & $\begin{array}{l}4.33 \\
0.96\end{array}$ & $\begin{array}{l}F_{w, j}{ }^{(3)}[R \mid R(\omega)] \\
F_{w, j}{ }^{(3)}[R \mid R(\omega)]\end{array}$ & $\begin{array}{r}0.40 \\
-0.03\end{array}$ \\
\hline \multicolumn{5}{|c|}{ Alcohols (22) } \\
\hline $\begin{aligned} & T_{r, b}>0.7(13) \\
& T_{r, b}>0.76 \\
& 0.7<T_{r, b}<0.76 \\
& T_{r, b}<0.7\end{aligned}$ & $\begin{array}{l}19.65 \\
15.85 \\
21.33 \\
8.93\end{array}$ & $\begin{array}{c}20.46 \\
15.86 \\
8.11 \\
17.59 \\
\text { or } \\
17.24\end{array}$ & $\begin{array}{c}F_{w, j}{ }^{(I)}[R \mid R(\omega)] \\
\mathrm{A}-\mathrm{W}\left(T_{b}\right) \\
F_{w, j}{ }^{(I)}[R \mid R(\omega)] \\
\mathrm{R} \\
\text { or } \\
F_{w, j}{ }^{(2)}[R \mid R(\omega)]\end{array}$ & $\begin{array}{c}0.81 \\
0.01 \\
-13.22 \\
8.66 \\
\text { or } \\
8.31\end{array}$ \\
\hline \multicolumn{5}{|c|}{ "Others" (41) } \\
\hline $\begin{array}{l}T_{r, b}>0.7(14) \\
T_{r, b}<0.7(27)\end{array}$ & $\begin{array}{l}0.49 \\
1.07 \\
0.81 \\
1.99 \\
\end{array}$ & $\begin{array}{l}0.66 \\
0.87 \\
0.86 \\
0.93 \\
\end{array}$ & $\begin{array}{c}\text { A-W } \\
\text { See breakdown below } \\
F_{w, j}{ }^{(3)}\left[A-W\left(T_{b}\right) \mid R(\omega)\right] \\
F_{w, j}[R(\omega)]\end{array}$ & $\begin{array}{c}0.17 \\
-0.20 \\
0.05 \\
-1.06 \\
\end{array}$ \\
\hline
\end{tabular}


However, for any given species, the slope of the $\log P_{v, r}$ vs. $T_{r}$ curve decreases as the reduced temperature increases, resulting in the normalized extrapolation burden decreasing, rather than increasing, as the reduced temperature of the lower known anchor point increases.

Consequently, when performing a species-by-species comparison of predictive error vs. extrapolation burden for methods with differing lower anchor points, the nonnormalized burden is more appropriate.

Such is the case in this study, for the lower known anchor point of the $F_{w, j}$ functions is not fixed but rather switches between the normal boiling point and the reduced temperature of 0.7. Hence, the non-normalized form is the more appropriate indicator of extrapolation burden when comparing the predictive performance of the recommended subset $F_{w, j}$ functions collectively with the source correlations. It is interesting to note that the subset recommended $F_{w, j}$ functions shown in Table 9 use the lowest reduced temperature of the two known vapor pressures $\left(P_{v, r, b}\right.$ and $P_{v, r, 0.7)}$ as the lowest anchor point for all but nine of the fiftyfour species. This is consistent with logic and previous results [17].

The modified Riedel correlation, $\mathrm{R}(\omega)$, by definition will always have the lowest reduced temperature of the two known anchor points. However, it was shown that $\mathrm{R}(\omega)$ does not always beat the other correlations in species head-to-head competition, nor does it produce less aggregate error for any species subset than the recommended $F_{w, j}$ function. This indicates that the improved predictability of the recommended $F_{w, j}$ functions results from more than just predominately using the lowest of the two known anchor points.

This can be seen in Figure 1, where the ratio of A\%Err is plotted vs. the ratio of the non-normalized burden relative to three source correlations, where the form of the ratio is Subset Recommended $F_{w, j}$ Functions : source correlation. One can see that the predictive error of the $F_{w, j}$ function relative to the $\mathrm{R}(\omega)$ correlation is less for the majority of the nine species where the $\Delta \log P_{v, r}$ for the $F_{w, j}$ function is larger than that of $\mathrm{R}(\omega)$. One can also see that the error of the $F_{w, j}$ function is less for the majority of the remaining species where the burden ratio is 1.0 (lowest anchor points are the same for both methods). Since the burden ratio is never less than one, the improved predictive capability can't be due to the $F_{w, j}$ function having a smaller extrapolation burden than $\mathrm{R}(\omega)$; rather, the error reduction is due to the prediction at the lowest of the four reduced temperatures used for the Wagner parameterizations of the recommended $F_{w, j}$ functions being accurate enough to essentially act as a new lower anchor point, reducing the effective non-normalized extrapolation burden below the value of the formal burden.

The Riedel plot in Figure 1 shows that the majority of the species have a burden ratio of one relative to the standard Riedel and yet an error ratio less than one, again suggesting that in such cases the lowest of the four reduced temperatures used for the recommended $F_{w, j}$ function essentially acts as an improved lower anchor point. All three plots in the figure reveal the impact of the accuracy of the lowest parameterization point used in the recommended $F_{w, j}$ function - the accuracy of the lowest parameterization point for the majority of species is sufficient to improve the predictive error at the normal fusion point.

\subsection{Comparison to $F_{w, j}[A A]$ Functions}

The results of the $F_{w, j}$ function using analytic predictions from Antoine constants has been presented elsewhere [18]; these functions were referred to as $F_{w, j}[A A]$, where the "AA" indicated that the four data points were Antoine analytics. Table 10 compares the average and incremental errors of the subset recommended predictive methods with those of the $F_{w, j}[A A]$ function for the 55 species used in that study. None of the alcohols had a normal boiling point reduced temperature above 0.76. Using analytic data from Antoine constants (a surrogate for limited-range experimental data) provides significantly better VLE prediction for the six alcohols with $T_{r, b}<0.7$ (cell shaded in gray) and has only slightly greater error for the seven alcohols with $T_{r, b}>0.7$. There is one caveat with the performance of the $F_{w, j}[A A]$ function for alcohols: the function failed the Waring test for three members of this subset - methanol $\left(T_{r, b}<0.7\right)$, and 1heptanol and 1-hexanol (both with $T_{r, b}>0.7$ ). Consequently, one may prefer to use the $F_{w, j}[A A]$ function for alcohols where $T_{r, b}<0.7$, unless the function fails the Waring test, in which case one could choose to use Riedel or the $F_{w, j}{ }^{(2)}[R \mid R(\omega)]$ function. The recommended predictive methods result in less error than the $F_{w, j}[A A]$ function for all the remaining substance subsets.

Summary statistics are provided in this paper for all results out of concern for brevity, but results for all individual species corresponding to Tables 3, 4, 6-9, and 10 can be found in Nichols [25], as can also a description of the calculation round off used for parameter inputs/outputs.

\section{Potential Modifications}

The results presented provide proof-of-principle that predictions from VLE correlations may be used to provide better predictive error near the triple point than the source correlations themselves. The three sets of four points used (summarized in Table 5) are chosen to illustrate the principle and are not meant to represent or suggest universal best fourpoint options. The Riedel and Ambrose-Walton are used as source correlations because of their general acceptance, applicability [5], and ease of use. However, developing predictive VLE correlations is an on-going focus of research in the literature, providing a wide array of possible source correlations, including multiple reference fluids, speciesspecific correction factors, and family-specific correlations.

Ambrose \& Patel [26] showed that two reference fluids could be used to predict vapor pressures better than one reference fluid. Sorner \& Strom [27] expanded to three reference fluids, and Rozhnov, Melnik, and Chmykhalo [28] developed an algorithm involving sixteen reference substances to predict vapor pressure for pure substances. One could add a species-specific correction factor to an existing correlation or methodology, similar to what Halm \& Stiel [22] attempted many years ago with their polar factor, or as An \& Yang [29] more recently have done for organics with the $\omega$-based Lee-Kesler equation. A predictive equation for a class or family of substances, such as that of Sanjari et al. [30] for refrigerants, could be used as a source correlation.

Although Waring [14] showed that a fourth parameter is necessary to accurately describe VLE over the entire two- 

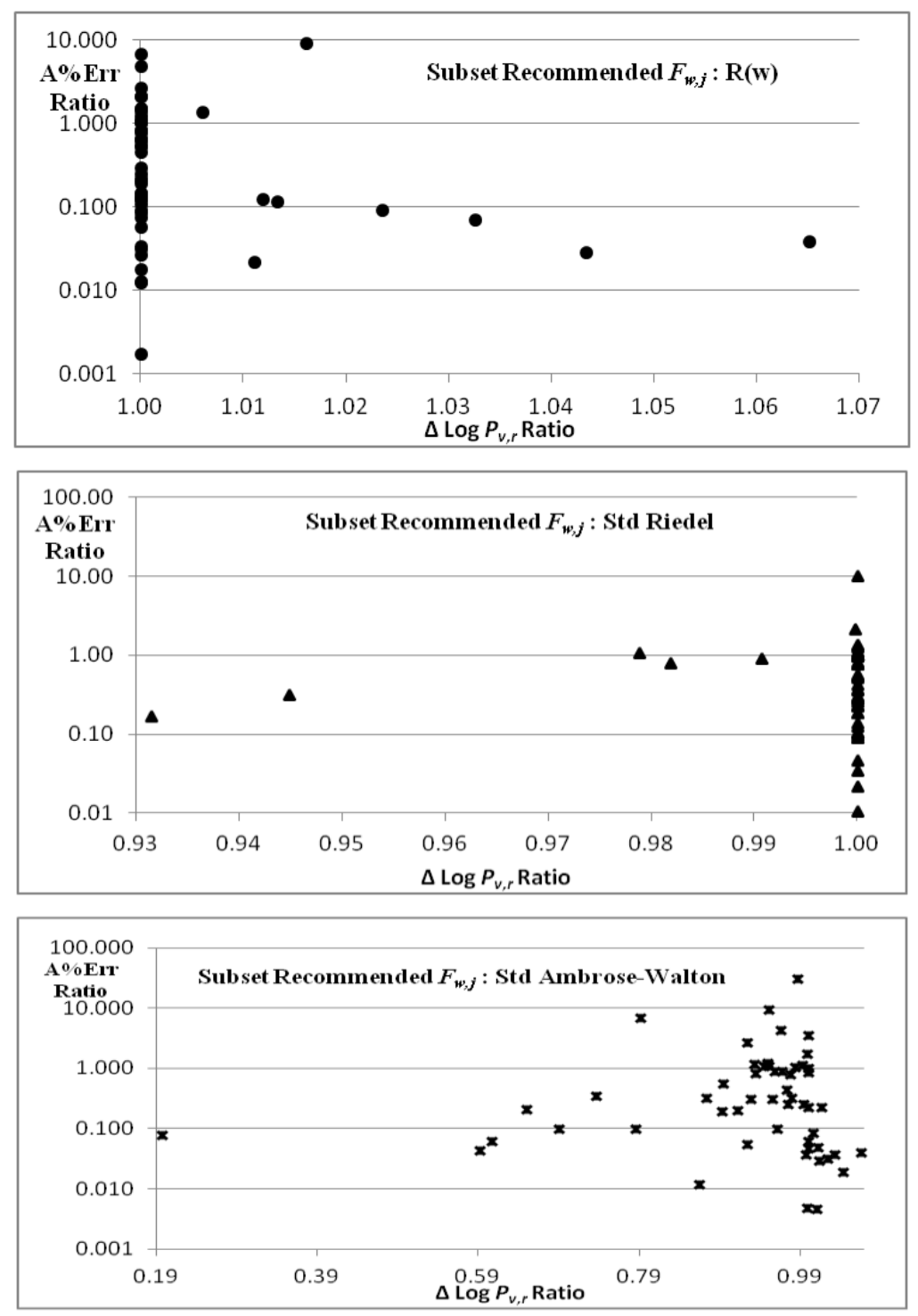

Figure 1. Ratio of A\%Err at $T_{r, f}$ vs. Ratio of Non-Normalized Extrapolation Burden for the Fifty-Four Species Corresponding to Subset Recommended $F_{w, j}$ Functions: $\bullet-F_{w, j}: R(\omega), \mathbf{\Delta}-F_{w, j}:$ Standard Riedel, * $-F_{w, j}:$ Standard Ambrose-Walton.

Table 10. Summary of Entire-Curve Incremental Errors for Recommended Predictive Methods vs. $F_{w, j}[A A]$ Functions. All Statistics Are for the Common 55 Species

\begin{tabular}{|c|c|c|c|c|c|c|}
\hline \multirow[b]{2}{*}{$T_{r, b}$ vs. 0.7} & \multirow{2}{*}{$\begin{array}{l}\text { Minimum Possible } \\
\text { Average A\%Err }\end{array}$} & \multicolumn{3}{|c|}{ Subset Predictive Recommendation } & \multicolumn{2}{|c|}{ Species-Specific $F_{w, j}[A A]$} \\
\hline & & Average A\%Err & Method & $\begin{array}{c}\text { Incremental } \\
\text { Error } \\
\end{array}$ & $\begin{array}{c}\text { Average } \\
\text { A\%Err }\end{array}$ & $\begin{array}{c}\text { Incremental } \\
\text { Error }\end{array}$ \\
\hline \multicolumn{7}{|c|}{ Organic Acids (6) } \\
\hline$T_{r, b}>0.7(4)$ & 3.69 & 5.46 & $F_{w, j}^{(3)}[R \mid R(\omega)]$ & 1.77 & 12.60 & 8.91 \\
\hline$T_{r, b}<0.7(2)$ & 0.98 & 0.95 & $F_{w, j}^{(3)}[R \mid R(\omega)]$ & -0.03 & 1.56 & 0.58 \\
\hline \multicolumn{7}{|c|}{ Alcohols (13) } \\
\hline$T_{r, b}>0.7(7)$ & 12.15 & 7.22 & $F_{w, j}^{(l)}[R \mid R(\omega)]$ & -4.93 & 7.41 & -4.74 \\
\hline$T_{r, b}>0.76(0)$ & N/A & N/A & $\mathrm{A}-\mathrm{W}\left(T_{b}\right)$ & N/A & N/A & N/A \\
\hline $0.7<T_{r, b}<0.76$ & 12.15 & 7.22 & $F_{w, j}^{(I)}[R \mid R(\omega)]$ & -4.93 & 7.41 & -4.74 \\
\hline & & 19.77 & $\mathrm{R}$ & 10.43 & & \\
\hline$T_{r, b}<0.7$ (6) & 9.35 & or & or & or & 13.14 & 3.79 \\
\hline & & 19.43 & $F_{w, j}^{(2)}[R \mid R(\omega)]$ & 10.08 & & \\
\hline \multicolumn{7}{|c|}{ "Others" (36) } \\
\hline$T_{r, b}>0.7(13)$ & 0.32 & 0.33 & $\mathrm{~A}-\mathrm{W}$ & 0.01 & 3.17 & 2.85 \\
\hline$T_{r, b}<0.7$ & 1.04 & 0.84 & See breakdown below & -0.20 & 2.07 & 1.03 \\
\hline Normal (17) & 0.71 & 0.85 & $F_{w, j}^{(3)}\left[A-W\left(T_{b}\right) \mid R(\omega)\right]$ & 0.14 & 1.60 & 0.89 \\
\hline Polar (6) & 1.99 & 0.80 & $F_{w, j}[R(\omega)]$ & -1.19 & 3.43 & 1.44 \\
\hline
\end{tabular}


phase curve, Vetere [31] concluded that the presence of the Wagner $\mathrm{d}$ constant is mostly inconsequential to predictive error when data of lower accuracy is used. He mathematically tied the values of the remaining three Wagner constants to the critical and normal boiling points, with the defining relationships dependent upon the species type. He concluded that the three-constant predictive Wagner equation was generally superior to other predictive correlations (including the original Riedel using $\mathrm{K}=0.0838$ ) while noting that it did perform significantly worse for a few species. Vetere [2], [20] subsequently attempted to improve the Riedel predictive method by making the $\mathrm{K}$ parameter dependent upon species type. Given that VLE from predictive correlations will generally be of lower accuracy relative to actual data, a potential modification to our approach would be to use predictive correlations to provide VLE inputs into the parameterization of a three-parameter Wagner equation rather than the full four-parameter form.

\section{Summary and Conclusions}

The $\mathrm{R}(\omega)$ and $\mathrm{A}-\mathrm{W}(\mathrm{Tb})$ correlations perform better than their standard counterparts in many cases (Table 4). The VLE behavior below and above the normal boiling point of organic acids, and of normal and non-alcohol polar species where $T_{r, b}<0.7$, can be described accurately on a consistent enough basis by the standard or modified Riedel and Ambrose-Walton correlations such that reasonable Wagner constants can be estimated from $F_{w, j}$ functions whose four points are taken from these predictive correlations. The VLE predictive error of such Wagner constants for the entire twophase curve approaches or beats the minimum predictive error possible (Tables $7 \& 9$ ). For all substance subsets, except the fourteen normal species with $T_{r, b}>0.7$, the subset best $F_{w, j}$ function results in less average error than the subset best correlation.

The correlation and prediction-based $F_{w, j}$ function recommended for the various substance subsets have predictive power superior to that of Wagner constants estimated from Antoine analytics, except for some alcohols (Table 10). This reinforces the previous conclusion [18] that solely using limited VLE data to extrapolate downward to the triple point provides an advantage primarily for species that are problematic for predictive correlations.

The possibility that merely four predictions from VLE correlations can be used to provide less predictive error than the source correlations themselves or less error than parameterizations using limited intervals of experimental data has been studied and proven. The fact that such error reduction is possible opens up new avenues for predictive improvement. Although complexity in an approach to VLE prediction is not a deterrent to a researcher, it can be to a field practitioner. There are many complex and family-specific VLE correlations in the literature - the possibility that only a few accurate predictions from such a correlation can be used to create a simple 4-parameter Wagner equation with equal or better predictive accuracy for the targeted species' VLE over the entire two-phase curve is of significant import to field practitioners. Instead of trying to incorporate several complex correlations for the pure-species domain into a multi-species VLE algorithm, for example, one could use a few points from each source correlation to algebraically generate a set of simpler, accurate Wagner equations to represent the pure-species domain.

The major benefit is for a species with sparse experimental data. The results presented here show that the source correlation does not have to be accurate at the triple point itself in order to parameterize Wagner constants better applicable for the entire two-phase curve. Rather, the source correlation needs to be accurate at a reduced temperature low enough to allow the effective extrapolation burden to be sufficiently reduced such that predictive capability towards the triple point is noticeably improved.

This work presents and demonstrates a technique to leverage VLE correlations more effectively, while lessening the dependency upon low-pressure thermodynamic data (VLE and/or thermal) for accurate parameterization of an entire-curve VLE equation such as Wagner's.

\section{Nomenclature}

$a, b, c, d \quad$ Wagner constants, Eq. (1)

$A^{+}, B^{+}, C^{+}, D^{+} \quad$ Parameters in Riedel

$f^{(0)}, f^{(1)}, f^{(2)} \quad$ Parameters in Ambrose-Walton

$F_{w, j} \quad$ Function used to estimate Wagner constants, Eq. (6).

$[A A]$ indicates calculations use Antoine analytic data.

Definition of other bracketed terms are given in text and summarized in Table 5.

$h$

Riedel equation parameter, Eq. (3g)

Parameter in the Riedel formulism.

For standard Riedel correlation, defined by Eqs. (3e) and (3f) for organic acids and alcohols, respectively, equal to 0.0838 otherwise.

For modified Riedel correlation, defined by Eq. (4b).

$K^{\prime} \quad$ Defined by Eq. (4c)

Ln Natural logarithm

$P \quad$ Pressure (bar)

$P_{c}:$ Critical pressure

$P_{v}$ : Vapor pressure

$P_{v, r}:$ Reduced vapor pressure (relative to critical point)

$P_{v, r, 0.7}$ : Reduced vapor pressure at reduced temperature of 0.7

$P_{v, r, b}:$ Reduced vapor pressure at normal boiling point

$P_{v, r, f}$ : Reduced vapor pressure at normal fusion point

$P_{v, r, S A}$ : Reduced vapor pressure of second anchor point in the modified Riedel correlation, which is chosen to be at $T_{r}=0.7$

$P_{v, r, T r i}$ : Reduced vapor pressure of data point "i" in the $F_{w, j}$ function, Eq. (6)

$Q \quad$ Parameter in Riedel formulism, Eq. (3b)

Thermodynamic temperature $(\mathrm{K})$

$T_{b}$ : Normal boiling point temperature

$T_{c}$ : Critical temperature

$T_{r}$ : Reduced temperature (relative to critical point)

$T_{r, f}:$ Reduced temperature of normal fusion point

$T_{r, S A}$ : Reduced temperature of second anchor point for modified Riedel correlation $\left(T_{r, S A}=0.7\right)$

Greek Letters

$\alpha_{c}$

$\eta_{j, i}$

$\tau$

$\Psi$

Parameter in Riedel formulism, Eq. (3c)

Coefficients in $F_{w, j}$ function, Eq. (6)

$1-T_{r}$

Parameter in Riedel formulism

$\Psi_{b}$ : Defined by Eq. (3d) for the standard Riedel correlation

$\Psi_{S A}$ : Defined by Eq. (4a) for the modified Riedel correlation

$\omega \quad$ Acentric factor

Subscripts

$i \quad$ Index notation for the four data points used by $F_{w, j}$ function and $\eta_{j, i}$ 
Index notation for the Wagner constant for $F_{w, j}$ function and $\eta_{j, i}$

Superscripts

(1)

$F_{w, j}$ function, data inputs pertain to reduced temperature point distribution of 0.6, 0.7, 0.8 and 0.9 $F_{w, j}$ function, data inputs pertain to reduced temperature point distribution of $0.3,0.6,0.7$ and 0.95

Abbreviations

$F_{w j}$ function, data inputs pertain to reduced

A\%Err Absolute value of percent error relative to the entirecurve Wagner analytic values

All $T_{r} \mathrm{~s} \quad$ Entire two-phase curve

A-W Correlation method that represents the standard Ambrose-Walton correlation

$\mathrm{A}-\mathrm{W}\left(T_{b}\right) \quad$ Correlation method that represents the modified Ambrose-Walton correlation

$\mathrm{R} \quad$ Correlation method that represents the standard Riedel correlation

$\mathrm{R}(\omega) \quad$ Correlation method that represents the modified Riedel correlation

Expressions

$\Delta \log P_{v, r} \quad$ Non-normalized extrapolation burden

$\Delta \log P_{v, r} / \Delta T_{r}$ Normalized extrapolation burden

\section{References}

[1] J. T. Wu, Z. G. Liu, "An Accurate Vapor Pressure Equation With Good Extrapolation Characteristics," Int. J. Thermophys., 26, 767-784, 2005.

[2] A. Vetere, "Again the Riedel equation," Fluid Phase Equilib., 240, 155-160, 2006.

[3] B. E. Poling, "Vapor pressure prediction from the triple point to the critical point," Fluid Phase Equilib., 116, 102109, 1996.

[4] D. Ambrose, "The Corelation And Estimation Of Vapour Pressures IV. Observations On Wagner's Method Of Fitting Equations To Vapour Pressures," J. Chem. Thermodyn., 18, 45-51, 1986.

[5] B. E. Poling, J. M. Prausnitz, and J. P. O'Connell, The Properties of Gases and Liquids, 5th ed. San Francisco, USA: Mc-Graw Hill, 2001.

[6] D. Ambrose, "The Correlation and Estimation of Vapour Pressures I . A Comparison of Three Vapour-Pressure Equations," J. Chem. Thermodyn., 10, 765-769, 1978.

[7] D. Ambrose, J. F. Counsell, C. P. Hicks, "Correlation And Estimation Of Vapor-Pressures II. New Procedure For Estimation And Extrapolation," J. Chem. Thermodyn., 10, 771-778, 1978.

[8] J. McGarry, "Correlation and Prediction of the Vapor Pressures of Pure Liquids over Large Pressure Ranges," Ind. Eng. Chem. Process Des. Dev., 22, 313-322, 1983.

[9] L. A. Forero, J. A. Velasquez J., "Wagner liquid - vapour pressure equation constants from a simple methodology," J. Chem. Thermodyn., 43, 1235-1251, 2011.

[10] D. Ambrose, R. H. Davies, "The Correlation And Estimation Of Vapor-Pressures III. Reference Values For Low-Pressure Estimations," J. Chem. Thermodyn., 12, 871-879, 1980.

[11] M. G. King and H. Al-Najjar, "Method For Correlating And Extending Vapor-Pressure Data To Lower Temperatures Using Thermal Data - Vapor-Pressure Equations For Some n-Alkanes At Temperatures Below Normal Boiling-Point," Chem. Eng. Sci., 29, 1003-1011, 1974.

[12] K. Ruzicka and V. Majer, "Simultaneous Treatment Of Vapor-Pressures And Related Thermal Data Between The Triple And Normal Boiling Temperatures For N-Alkanes C5-C20," J. Phys. Chem. Ref. Data, 23, 1-39, 1994.

[13] G. Thodos, "Vapor Pressures Of Normal Saturated
Hydrocarbons," Ind. Eng. Chem., 42, 1514-1526, 1950.

[14] W. Waring, "Form Of A Wide-Range Vapor Pressure Equation," Ind. Eng. Chem., 46, 762-763, 1954.

[15] D. Ambrose and N. B. Ghiassee, "Vapour Pressures And Critical Temperatures And Critical Pressures Of Some Alkanoic Acids: C1 to C10," J. Chem. Thermodyn., 19, 505-519, 1987.

[16] S. Velasco, J. A. White, K. Srinivasan, and P. Dutta, "Waring and Riedel Functions for the Liquid-Vapor Coexistence Curve," Ind. Eng. Chem. Res., 51, 3197-3202, 2012.

[17] T. T. Nichols and V. P. Utgikar, "Wagner Equation Predicting Entire Curve for Pure Fluids from Limited VLE Data: Error Dependency Upon Data Interval \& FullyDetermined Case," Int. J. Thermodyn., 21, 38-53, 2018.

[18] T. T. Nichols and V. P. Utgikar, "Wagner Equation Predicting Entire Curve for Pure Fluids from Limited VLE Data: Critical Point and Four Antoine Analytic Points," Fluid Phase Equilib., 460, 1-16, 2018.

[19] D. Ambrose, J. Walton, "Vapor-Pressures Up To Their Critical-Temperatures Of Normal Alkanes And 1Alkanols," Pure Appl. Chem., 61, 1395-1403, 1989.

[20] A. Vetere, "The Riedel Equation," Ind. Eng. Chem. Res., 30, 2487-2492, 1991.

[21] E.W. Lemmon, M.O. McLinden, D.G. Friend, "Thermophysical Properties Of Fluid Systems," NIST Chemistry WebBook, NIST Standard Reference Database Number 69, Eds. P.J. Linstrom and W.G. Mallard, National Institute of Standards and Technology, Gaithersburg MD, 20899, 2011. Available: http://webbook.nist.gov. [Accessed: 1-Sep-2011].

[22] R. L. Halm, L. I. Stiel, "A Fourth Parameter For The Vapor Pressure And Entropy Of Vaporization Of Polar Fluids," AIChE J., 13, 351-355, 1967.

[23] R. L. Halm and L. I. Stiel, "Saturated-Liquid And Vapor Densities For Polar Fluids," AIChE J., 16, 3-8, 1970.

[24] G. Z. A. Wu, L. I. Stiel, "A Generalized Equation Of State For The Thermodynamic Properties Of Polar Fluids," AIChE J., 31, 1632-1644, 1985.

[25] T. T. Nichols, "Predicting Vapor-Liquid Equilibrium Over the Entire Two-Phase Region Using Wagner Equation with Only Limited VLE Data," Ph.D. Dissertation, University of Idaho, 2017.

[26] D. Ambrose, N. C. Patel, "The Correlation And Estimation Of Vapour Pressures IV. Extrapolation Of Vapour Pressures And Estimation Of Critical Pressures By The Principle Of Corresponding States Using Two Reference Fluids With Non-Spherical Molecules," J. Chem. Thermodyn., 16, 459-468, 1984.

[27] M. Sorner, K. Strom, “A Proposal For Two New Parameters Based On Shape/Size And Polarity, To Be Used In A Four-Parameter Corresponding States Expression," Chem. Eng. Commun., 189, 1447-1469, 2002.

[28] M. S. Rozhnov, D. N. Mel'nik, P. A. Chmykhalo, “A Generalized Equation For The Saturation Vapor Pressure Of Individual Substances," Meas. Tech., 51, 755-761, 2008.

[29] H. An W. Yang, "A New Generalized Correlation for Accurate Vapor Pressure Prediction," Chem. Phys. Lett., 543, 188-192, 2012.

[30] E. Sanjari, M. Honarmand, H. Badihi, A. Ghaheri, “An Accurate Generalized Model for Predict Vapor Pressure of Refrigerants," Int. J. Refrig., 36, 1327-1332, 2013.

[31] A. Vetere, "Predicting the Vapor Pressures of Pure Compounds by Using the Wagner Equation," Fluid Phase Equilib., 62, 1-10, 1991. 